\title{
Filling a Multicolor Urn: an axiomatic analysis
}

\section{Hervé Moulin and Richard Stong}

\author{
Rice University
}

January 2001 


\begin{abstract}
We study the probabilistic distribution of identical successive units. We represent the allocation process as the filling of an urn with balls of different colors (one color per agent). Applications include the scheduling of homogeneous tasks among workers and allocating new workers between divisions. The fixed chances methods allocate each unit independently of the current distribution of shares. The Polya-Eggenberger methods place in an urn a fixed number of balls and draw from the urn with replacement of two balls of the color drawn.

These two families of urn-filling methods emerge uniquely from our axiomatic discussion involving: a version of the familiar Con-

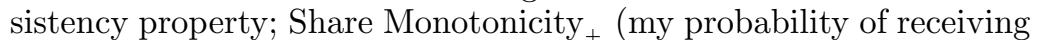
the next ball is non-decreasing in my current share); Independence of Transfers (transferring balls across agents is not profitable) and Order Independence (a sequence of successive allocations is as likely as any permuted sequence).

We also explore the impact of Share Monotonicity_ (my probability of receiving the next ball is non-increasing in my current share), leading to an equalization of individual shares along a fixed standard of comparison.
\end{abstract}

Keywords: urn-filling methods, markovian, consistency, Polya-Eggenberger

Hervé Moulin, Department of Economics, MS 22, Rice University, P.O. Box 1892, Houston, TX 77251-1892, USA, moulin@rice.edu, http://www.ruf.rice.edu/ econ/papers/index.html

Richard Stong, Department of Mathematics, MS 136, Rice University, P.O. Box 1892, Houston, TX 77251-1892, USA, stong @ rice.edu

JEL: D63

This research is supported by the NSF under grant SES0096230.

We gratefully acknowledge the critical comments of seminar and conference participants in Penn State, Johns Hopkins, UT Austin, SMU, Alicante (Social Choice and Welfare meetings), and Bilbao (Game Theory meetings). 


\section{Filling a Multicolor Urn: an axiomatic analysis}

\section{Introduction}

In an urn-filling problem, there is a set $N=\{1,2, \ldots, n\}$ of agents, each agent $i$ being represented by a different color. The urn contains initially $x_{i}$ balls of color $i$, and $t$ additional balls must be added to the urn. The question is to allocate the $t$ balls between the agents in $N$, namely to paint each ball in one of the $n$ colors. Lotteries are used for the sake of fairness: e.g., if $t=1$, the single ball may be equally divided among the $n$ agents by assigning it to each agent $i$ with probability $1 / n$.

An urn-filling method selects a (probabilistic) allocation for each urn-filling problem.

The urn-filling model represents a variety of distributive justice problems where a given set of agents share some homogeneous indivisible units. For instance the $i-t h$ department of a university college currently has $x_{i}$ positions and $t$ new positions have been funded, that must be allocated between the departments. Or agent $i$ represents a community (city, region), $x_{i}$ is the number of recruits that have been drafted in community $i$, and an additional burden of $t$ draftees must be divided among the communities. Or the agents share a house, $x_{i}$ is the number of times housemate $i$ has performed a certain chore (clean the yard), and the issue is who will be next.

In the above examples, $x_{i}$ represents agent $i$ 's current share of resources, but many other interpretations are possible. For instance $x_{i}$ could be the number of recruits that community $i$ offers to draft, and the total draft burden is $t+\sum_{i} x_{i}$. Or $x_{i}$ is the number of new classes that department $i$ wishes to offer, and the demand for $t+\sum_{i} x_{i}$ classes must be met, or community $i$ offers to host $x_{i}$ refugees, but the total number of refugees is $t+\sum_{i} x_{i}$. And so on.

The extreme simplicity of our model comes from the fact that agents are only differentiated by a single parameter $x_{i}$, a non negative integer that can be variously interpreted as an actual share of the resources, a demand, a claim, a liability and so on. An urn-filling method associates to each profile $\left(x_{1}, \ldots, x_{n}\right)$ and to each (positive integer) $t$, a probability distribution over all allocations of $t$ units among $n$ agents.

Our main axioms are two invariance properties linking the solutions to different urn-filling problems. The Markovian property says that the allocation of $t$ units is equivalent to $t$ successive independent allocations of one unit: in other words the method is entirely determined by the way it allocates a single unit in the urn $\left(x_{1}, \ldots, x_{n}\right)$, i.e., by the probabilistic distribution over $N$ describing who receives the next unit given the current state of the urn. The Consistency property says that the allocation of $t$ units to $n$ agents is equivalent to $n$ successive independent allocations to the individual agents. That is, we choose first a 
realization $y_{1}$ of agent 1's share, then a realization $y_{2}$ of agent 2's share when $t-y_{1}$ units are allocated among $N \backslash\{1\}$; next $y_{3}$ realizes agent 3's share from $t-y_{1}-y_{2}$ units among $N \backslash\{1,2\}$, and so on.

Obviously the Markovian and Consistency properties have much mathematical bite, without conveying any notion of interpersonal equity: for instance the urn-filling method that always gives all $t$ units to the same agent $i^{*}$ (the "dictator") is both markovian and consistent, as is the (egalitarian) method that allocates each successive unit with equal probability $1 / n$ to each agent. If the entire family of markovian and consistent methods is too complicated for a compact description, we find that an additional mild monotonicity requirement points to the simple and natural Polya-Eggenberger urn-filling methods (see, e.g., Johnson and Kotz [1977]).

In a Polya-Eggenberger method, the urn contains a fixed number $\lambda_{i}$ of balls of color $i$ plus agent $i^{\prime} s$ current share $x_{i}$. The next unit is allocated by drawing a ball from the urn (containing $\sum_{i}\left(\lambda_{i}+x_{i}\right)$ balls) and replacing it with two balls of the same color. Thus agent $i$ receives this unit with probability proportional to $\lambda_{i}+x_{i}$. Note that $\lambda_{i}$ is a positive real number, not necessarily an integer. In such a method, the greater agent $i^{\prime} s$ current share $x_{i}$, the greater his chances of receiving the next unit. One of our main results (Theorem 1), describes the family of markovian and consistent urn-filling methods sharing this monotonicity property (a higher current share $x_{i}$ means a higher chance of receiving the next unit). The family consists, essentially, of the Polya-Eggenberger methods just described, and of the fixed chances methods, where the probability distribution of the next unit is altogether independent of the current content of the urn.

Our second main result (Theorem 2) characterizes the markovian and consistent urn-filling methods with the opposite monotonicity property: a higher current share $x_{i}$ implies a lower chance of receiving the next unit. These include the fixed chances methods, and many other methods that tend to equalize shares just like the P.E. methods tend to spread them.

Before announcing the contents of the paper, we review the relevant literature.

The seminal model is the "rationing according to claims" ' problem introduced by O'Neill [1982], and further discussed by Aumann and Maschler [1985], Young [1987] [1988] and many others under various names, such as bankruptcy or inheritance problem (Thomson [1995] offers a survey). In the rationing model, $x_{i}$ is a positive real number representing agent i's claim/demand/liability, and the available resources $t^{\prime}$, also a positive real number, fall short of the total claim: $t^{\prime}<\sum_{i} x_{i}$. There is also a dual (and less studied) "surplus-sharing" model where the resources $t^{\prime}$ exceed the sum of individual claims, $t^{\prime}>\sum_{i} x_{i}$ : Young [1987], Moulin [1987], Chun [1989], Herrero et al. [1999].

The model of this paper belongs to the surplus-sharing family (modulo the change of variable $t=t^{\prime}-\sum_{i} x_{i}$ ), with two special features of crucial technical importance. First the homogeneous commodity comes in indivisible units, second the method selects a lottery over the feasible deterministic allocations.

Our two key axioms, Consistency and the Markovian property, are familiar to the rationing and surplus-sharing literatures. Consistency has played the 
leading role, ever since the characterization of the symmetric and consistent rationing or surplus-sharing methods in the classical model (i.e., divisible good and deterministic shares) by Young $[1987]^{1}$. These methods all have a parametric representation, which appears to have no counterpart in the probabilistic model of this paper.

The Markovian property plays a smaller role than Consistency in the classical literature, yet it appears prominently (under various names) in Moulin [1987], Young [1988], Moulin [2000a], Herrero et al. [1999].

In the classical model, a full characterization of all markovian and consistent methods is still out of reach ${ }^{2}$. A considerable advantage of the probabilistic model is that, upon adding mild monotonicity or individual rationality conditions, we get a full description of the impact of these two axioms.

Two recent papers by the same authors, Moulin [2000b], and Moulin and Stong [2000], are a key inspiration to the present work. In the same probabilistic model with indivisible homogeneous goods, these papers consider the rationing problem, namely the total resources $t^{\prime}$ fall short of the total claim $\sum_{i} x_{i}$. A solution to the rationing problem is a method to empty from the urn $\left(x_{1}, \ldots, x_{n}\right)$ $t=\sum_{i} x_{i}-t^{\prime}$ units. The question is which should be the color of the balls we take out of the urn? From the same consistency and Markovian axioms, as well as similar monotonicity and individual rationality constraints, the method called proportional emerges: this method simply empties the urn without replacement, picking the next unit away from agent $i$ with probability $x_{i} / \sum_{j} x_{j}$. Thus the single proportional urn-emptying method is the counterpart of the family of the Polya-Eggenberger urn-filling methods. After each one of our main result, we comment on the corresponding result, if any, in the urn-emptying model: see Remarks 2 to 6 . We also stress that many proof techniques in the Appendices 1 to 6 follow closely those of the companion papers.

\section{Overview of the paper}

The model and the key axioms are defined in Section 2. They include Consistency and the Markovian property, as well as the two properties mentioned above called Share Monotonicity + and Share Monotonicity $\left(S M_{+}\right.$and $\left.S M_{-}\right)$, stating respectively that agent $i^{\prime}$ s share of the surplus $t$ does not decrease, or does not increase, as agent $i^{\prime}$ s share $x_{i}$ increases. Each assumption is compelling in certain contexts: $S M_{+}$in the draft or academic recruitment example, where a larger share reflects a larger liability or a larger claim in the next units, $S M_{-}$ in the chores examples, where a larger record of chores in the past warrants a lower contribution tomorrow.

In Section 3, we restrict attention to deterministic markovian and consistent methods. A simple description of all such methods is not possible, even in this simple case. Yet the subset of those methods satisfying Share Monotonicity $\varepsilon, \varepsilon=$ ,+- has a very simple structure. Under $S M_{+}$we are left with the unappealing "winner takes all" methods whereby any urn will be filled exclusively to the

\footnotetext{
${ }^{1}$ Young's result also assumes some continuity and monotonicity properties.

${ }^{2}$ Young [1987] adds a symmetry assumption plus some regularity properties, and Moulin [2000a] adds an equally powerful "dual" markovian property.
} 
benefit of one agent, though which agent is the "winner" may depend upon the initial urn. Under $S M_{-}$we find the plausible equalizing methods that essentially fill the urn according to a fixed pattern.

The Polya-Eggenberger (P.E.) methods are defined in Section 4, and characterized in Theorem 1, together with the fixed chances methods (where the distribution of the next unit does not depend on the urn at all). Theorem 1 uses four axioms: Consistency, the Markovian property, Share Monotonicity+ and a mild individual rationality property stating that any agent stands some positive chance of receiving some unit sometime in the future. The latter axiom is called Positive Future Shares (PFS).

Theorem 2 in Section 5 describes the urn-filling methods meeting the same four axioms, except that Share Monotonicity + is replaced by Share Monotonicity . We find a rich family of methods patching together an equalizing method as in Section 3, at most one fixed chances method, as well as some pieces of a proportional filling method.

In Section 6, we introduce two additional axioms. The first one, Invariance under Transfers, is an old friend of the rationing and surplus-sharing literatures in the classical model (Banker [1981], Moulin [1987]). It states that interpersonal transfers of shares (from $x_{1}, x_{2}$ to $x_{1}+3, x_{2}-3$, say) are never profitable (the total share of agents 1 and 2 will not change). The second axiom, Order Independence, is new, and applies to markovian methods only. Think of allocating $t$ units as a sequence of length $t$ describing who receives each successive unit; the OI axiom requires that two realizations of the sequence that sum up to the same profile of shares, be equiprobable. Thus OI conveys the idea that the filling method is chronologically unbiased: it schedules agents equally often in the early rounds or in the late ones. In probability theory, OI corresponds to the property of random processes called exchangeability (Billingsley [1995]).

Theorem 3 in Section 6 shows that the only markovian filling methods meeting IT and OI are, once again, the Polya-Eggenberger and the fixed chances methods.

Section 8 focuses on the markovian methods meeting OI but not necessarily consistent. These methods are interesting in their own right thanks to an appealing bayesian interpretation: Theorem 4 .

The six Appendices contain all the proofs as well as several extensions and variants of the four main results. Specifically, we consider in Appendix 2 what happens to Theorem 1 when we drop the Positive Future Shares requirement. In Appendix 5 we give a characterization, albeit a non-intuitive one, of all markovian and consistent methods. Finally Appendix 6 offers a complete description of the markovian and consistent methods also invariant under transfers.

\section{Urn-filling methods and the key axioms}

Let $\mathbb{N}=\{0,1,2, \ldots\}$ be the set of natural integers and $N$ be a finite set of agents. An urn is a pair $(N, x)$ where $x=\left(x_{i}\right)_{i \in N}$ is a vector in $\mathbb{N}^{N}$, of which the $i-t h$ 
component $x_{i}$ represent agent $i^{\prime}$ s allocation. For each subset $S$ of $N$, we write $\sum_{i \in S} x_{i}=x_{S}$.

A filling method $r$ specifies for each non-empty subset $N$ of a given (finite or infinite) set $\mathcal{N}$ of potential agents, for each initial urn $(N, x)$ and each positive integer $t$, a random variable $r(N, x, t)=Y=\left(Y_{i}\right)_{i \in N}$, where $Y_{i}$ is integer-valued, $Y_{i} \geq x_{i}$, and $\sum_{i} Y_{i}=x_{N}+t$.

We impose two powerful decomposition properties on filling methods: the Markovian property makes the addition of $t$ units equivalent to $t$ independent successive additions of one unit; the consistency property makes the filling of an $N$-urn equivalent to first computing agent $i^{\prime}$ s (random) share, then computing independently the allocation of the remaining units among the remaining agents $N \backslash i$. The former property links the association of successive units, the latter links the allocation across nested subsets of agents.

Markovian Property(M): for all $N, t, x, y$ s.t. $y_{N}=x_{N}+t$ and $y \geq x$

$$
\begin{aligned}
\operatorname{proba}\{r(x, t) & =y\}= \\
\sum_{i \in N} \operatorname{proba}\{r(x, 1) & \left.=x+e_{i}\right\} \cdot \operatorname{proba}\left\{r\left(x+e_{i}, t-1\right)=y\right\}
\end{aligned}
$$

where $e_{i}$ is the $i$-th coordinate in $\mathbb{N}^{N}$, and where we set proba $\left\{r\left(x+e_{i}, t-1=\right.\right.$ $y\}=0$ if $y_{i}=x_{i}$ (in which case filling the urn from $x$ to $y$ precludes giving any unit to agent $i$ ). Note that we omit from equation (1) any reference to $\mathcal{N}$ since $N$ plays no role in equation (1).

Starting from an arbitrary urn $(N, x)$, suppose we add one unit according to $r$, so that the content of the urn becomes $r(N, x, 1)=x^{1}$, next we add one unit to the (realization of) urn $x^{1}$, that becomes $r\left(N, x^{1}, 1\right)=x^{2}$ (this new draw being independent of the first), and keep adding units in same fashion. The Markovian property says that $t$ iterations of this algorithm generate a random variable $Y$ with precisely the same probability distribution as $r(N, x, t)$. Therefore a markovian filling mechanism is entirely described by a mapping $(N, x) \rightarrow p(N, x)$, specifying for each urn $(N, x)$ the probability distribution of $r(N, x, 1): p_{i}(N, x)$ is the probability that agent $i$ receives the first unit, or

$$
p_{i}(N, x)=\operatorname{proba}\left\{r(N, x, 1)=x+e_{i}\right\} .
$$

Consistency (CSY): for all $N, t, x, i \in N$, and $y, y \geq x$ and $y_{N}=x_{N}+t$

$$
\begin{aligned}
\operatorname{proba}\{r(N, x, t) & =y\}= \\
\operatorname{proba}\left\{r_{i}(N, x, t)\right. & \left.=y_{i}\right\} \cdot \operatorname{proba}\left\{r\left(N \backslash i, x_{-i}, t-y_{i}+x_{i}\right)=y_{-i}\right\}
\end{aligned}
$$

where $x_{-i}, y_{-i}$ are the projections of $x, y$ on $\mathbb{N}^{N \backslash i}$.

Adding $t$ units to the urn $x$ can be decomposed into two stochastically independent moves, the first one choosing agent $i$ 's share $y_{i}$ (according to the 
probability distribution of $\left.r_{i}(N, x, t)\right)$ and the second one adding $t-y_{i}$ units to the urn $\left(N \backslash i, x_{-i}\right)$, no longer paying attention to agent $i$.

We give first some examples of filling methods failing one or the other of the two basic requirements. We set $n=|N|$. A non markovian example is the random dictator method:

$$
\text { for all } N, t, x \text { and } i: \operatorname{proba}\left\{r(N, x, t)=x+t e_{i}\right\}=\frac{1}{n}
$$

Note that random dictator is consistent, however.

An easy way to generate a non consistent method is to let one type of method govern the filling of urns among three agents, and a completely different type for filling urns with two agents. One instance is the markovian method:

$$
\begin{aligned}
p_{i}(N, x) & =\frac{1}{n} \text { for all } N, x \text { and } i \text { whenever }|N| \geq 3 \\
p_{i}(\{i, j\}, x) & =1 \text { if } x_{i}<x_{j} ;=\frac{1}{2} \text { if } x_{i}=x_{j} ;=0 \text { if } x_{i}>x_{j}
\end{aligned}
$$

The family of markovian and consistent filling methods is still very complicated (see Appendix 5), and in order to provide an easy description, we shall add one of two natural, and mild, monotonicity properties.

When agent $i^{\prime}$ s share in the urn $(N, x)$ increases from $x_{i}$ to $x_{i}+1$, does his share of a given number of additional units go up or down? If the units being allocated represent the dividends of an investment and $x_{i}$ represent agent $i^{\prime}$ s share in total investment $x_{N}$, it is natural to require that the larger $x_{i}$ is, the larger agent $i$ 's share of the dividends. Similarly, if $x_{i}$ represent agent $i$ 's tax contributions, and the units are an additional tax to be levied, a larger tax payment yesterday warrants a higher share of the tax burden today.

On the other hand if units represent chores (washing the dishes, sending recruits to be drafted), a higher number of chores performed so far is grounds to receive a lesser share of the new chores to be divided.

These examples suggest the following two monotonicity properties, pulling the filling method in opposite directions:

$$
\begin{aligned}
\text { Share Monotonicity }{ }_{+}\left(S M_{+}\right) & : \quad \text { for all } N, x, t \text { and } i \\
r_{i}(N, x, t)-x_{i} & \leq r_{i}\left(N, x+e_{i}, t\right)-\left(x_{i}+1\right)
\end{aligned}
$$

where the inequality between random variable means stochastic dominance.

$$
\begin{aligned}
\text { Share Monotonicity_ }\left(S M_{-}\right) & : \quad \text { for all } N, x, t \text { and } i \\
r_{i}(N, x, t)-x_{i} & \geq r_{i}\left(N, x+e_{i}, t\right)-\left(x_{i}+1\right)
\end{aligned}
$$

The property $S M_{-}$conveys the idea of a feedback equalizing individual shares, in the sense that a higher share is less likely to grow than a smaller 
one. Symmetrically, $S M_{+}$suggests a reinforcement mechanism whereby a larger share is growing faster than a smaller one: success breeds success and it is not inconceivable that one agent's share overtakes (almost) the entire urn ${ }^{3}$.

Notice that $S M_{+}$and $S M_{-}$express a certain notion of fairness, but do not include any element of interpersonal comparison of shares. Both axioms are, in fact, compatible with any amount of asymmetry in the treatment of the different agents. For instance consider a fixed chances filling method, namely a markovian method where $p(N, x)=\alpha$ is independent of $x$. This method meets both $S M_{+}$and $S M_{-}$. As $\alpha$ varies in the $N-$ simplex, it embodies an arbitrary amount of interpersonal discrimination.

Remark 1 . In our characterization results (such as Theorems 1, 2 and Proposition1) we only need to assume a weaker property than $S M_{+,-}$, namely the special case $t=1$ in inequalities (5) or (6):

$$
\begin{aligned}
& S M_{+}^{1} \quad: \quad p_{i}(N, x) \text { is non decreasing in } x_{i} \\
& S M_{-}^{1} \quad: \quad p_{i}(N, x) \text { is non increasing in } x_{i}
\end{aligned}
$$

When $N$ contains three or more agents, the $S M_{\varepsilon}^{1}$ property, for $\varepsilon=+,-$, is strictly weaker than $S M_{\varepsilon}$ as the reader can easily verify ${ }^{4}$.

Remark 2 An urn-emptying method $r$ specifies for each initial urn $(N, x)$ and each integer $t, 1 \leq t \leq x_{N}$, a random variable $r(N, x, t)=Y$ where $Y_{i}$ is integervalued, $0 \leq Y_{i} \leq x_{i}$, and $\sum_{i} Y_{i}=x_{N}-t$. One interpretation is that $x_{i}$ is agent $i$ 's claim, or demand, and $t$ is the size of the deficit, namely the difference between total demand and actual resources. Another familiar interpretation is taxation: $x_{i}$ represents agent $i^{\prime}$ s tax liability and $t$ is the total tax to be levied. See the discussion of the rationing/taxation model in Section 1.

The two companion papers Moulin [2000b] and Moulin and Stong [2000] discusses the urn-emptying model, as well as the "dual"problem of filling an urn with a cap, namely allocating $t$ units among $N$ when agent $i^{\prime} s$ demand $x_{i}$ is an upper bound on his (random) share $Y_{i}$. The main axioms used in Moulin and Stong [2000] are precisely the same as in the current paper, namely Consistency, the Markovian property (under the name of Upper Composition), and Share Monotonicity $_{+}$(under the name of Demand Monotonicity*), namely a larger demand implies a larger responsibility in the deficit:

$$
x_{i}-r_{i}(N, x, t) \leq\left(x_{i}+1\right)-r_{i}\left(N, x+e_{i}, t\right)
$$

\footnotetext{
${ }^{3}$ This however, does not happen in the Polya-Eggenberger filling methods discussed from Section 4 onward.

${ }^{4}$ The case $|N|=2$ is an exception. There the two properties are equivalent, as can be shown by mimicking the proof of Lemma 4 in Moulin and Stong [2000].
} 
Only the Share Monotonicity_ axiom has no analog in the urn-emptying model, as the inequality $r_{i}(x, t)+1 \leq r_{i}\left(x+e_{i}, t\right)$ for all $x, t$ would imply $r_{i}(x, t) \geq$ $r_{i}\left(\left(x_{i}-1, x_{-i}\right), t\right)+1 \geq r_{i}\left(\left(x_{i}-2, x_{-i}\right), t\right)+2 \geq \ldots \geq x_{i}$, contradiction.

Most of the results discussed below have a counterpart in the urn-emptying model, in some cases an almost identical statement. See Remarks 3, 4, 5 and 6. The proof techniques of Proposition 1, Theorems 1 and 2 are adapted from those in the urn-emptying companion paper.

\section{The case of deterministic methods}

In this section we restrict attention to deterministic methods, namely $r(N, t, x)$ is not random after all. The set of markovian and consistent methods meeting one of the two monotonicity axioms is both easy to describe and normatively unappealing: e.g., in the case of $S M_{+}$, these methods leave no room for a fair compromise.

The probability distribution $p(N, x)$ is concentrated on one of the $n$ coordinate vectors, so the set $\mathbb{N}^{N}$ is partitioned into $n$ (possibly empty) sets $M_{i}$, with $x \in M_{i}$ iff $p(N, x)=e_{i}$. The axiom $S M_{+}^{1}$ says $x \in M_{i} \Rightarrow x+e_{i} \in M_{i}:$ if the filling method gives the first unit to agent $i$ when the urn content is $x$, then agent $i$ receives all subsequent units. Symmetrically, $S M_{-}^{1}$ says $x \in M_{i} \Rightarrow x-e_{i} \in M_{i}$ : if agent $i$ receives the first unit at urn $x$, she is also the first to be served whenever her share is smaller.

Definition $1 A$ (deterministic) standard of comparison is a linear ordering $\succ$ of $\mathcal{N} \times \mathbb{N}$, increasing in the second coordinate: $\left(i, x_{i}\right) \prec\left(i, x_{i}+1\right)$ for all $\left(i, x_{i}\right) \in$ $\mathcal{N} \times \mathbb{N}$. The $\succ$-winner-takes-all method is the markovian filling method:

$$
p(N, x)=e_{i} \Leftrightarrow\left(i, x_{i}\right) \succ\left(j, x_{j}\right) \text { for all } j \in N \backslash i
$$

The $\succ$-equalizing method is the markovian filling method:

$$
p(N, x)=e_{i} \Leftrightarrow\left(j, x_{j}\right) \succ\left(i, x_{i}\right) \text { for all } j \in N \backslash i
$$

For instance, let $N=\{1,2, \ldots, n\}$ and $\succ$ be the almost symmetric standard:

$$
\left(i, x_{i}\right) \succ\left(j, x_{j}\right) \text { iff }\left\{x_{i}>x_{j}\right\} \text { or }\left\{x_{i}=x_{j} \text { and } i<j\right\}
$$

Starting from an urn $\left(x_{1}, \ldots, x_{n}\right)$ the winner-takes-all method gives all units to an agent with the largest share, whereas the equalizing method gives the next unit to an agent with the smallest share. If $\mathcal{N}$ contains only two agents, a markovian filling method meeting $S M_{+}^{1}$ (resp. $S M_{-}^{1}$ ) is the winner-takesall (resp. equalizing) method associated with the following standard: $\left(i, x_{i}\right) \succ$ $\left(j, x_{j}\right)$ iff $p(x)=e_{i}$. In order to generalize this statement to an arbitrary, finite 
or infinite, set $\mathcal{N}$ we need the Consistency property, which in the deterministic case reads as follows:

$$
r_{j}(N, x, t)=r_{j}\left(N \backslash i, x_{-i}, t-r_{i}(N, x, t)+x_{i}\right) \text { for all } N, x, t, i \text { and } j, i \neq j
$$

If agent $i$ 's share is $y_{i}$ when $t$ units are shared among the agents in $N$ with claim profile $x$, then the allocation of $t-y_{i}$ units among $N \backslash i$ with claims $x_{-i}$ matches the allocation of $t$ units among $N$.

Proposition 1 A deterministic filling method is markovian (1), consistent (2) and share monotonic . $_{+}$(5), if and only if it is the $\succ$-winner-takes-all method associated with a standard of comparison $\succ$.

A deterministic method is markovian, consistent and share monotonic_(6), if and only if it is the $\succ$-equalizing method for a standard of comparison $\succ$.

Note that it is enough to assume the weak form $S M_{\varepsilon}^{1}$ of Share Monotonicity $\varepsilon$ (see Remark 1) for the "only if" statement.

Remark 3 In the case of urn-emptying methods (Remark 2), Proposition $1^{*}$ in Moulin and Stong [2000] describes the set of methods characterized by the Markovian property, Consistency and Share Monotonicity ${ }_{+}$(see Remark 2).

These methods are the direct counterpart of the equalizing methods in Proposition 1. For a standard $\succ$, i.e., an ordering of $\mathcal{N} \times \mathbb{N}$ increasing in the second coordinate, the first unit of deficit is taken away from $x_{i}$ iff $\left(i, x_{i}\right)$ is the largest claim:

$$
r(N, x, 1)=x-e_{i} \Leftrightarrow\left(i, x_{i}\right) \succ\left(j, x_{j}\right) \text { for all } j \neq i
$$

As the urn empties, this method equalizes the claims $\left(i, x_{i}\right)$ with respect to the standard $\succ$.

\section{Polya-Eggenberger filling methods}

Definition 2 Choose for each agent $i$ in $\mathcal{N}$ a positive weight $\lambda_{i}-$ a positive real number - and denote by $\lambda$ the $\mathcal{N}$-profile of these weights. The $\lambda$-Polya-Eggenberger filling method is the following markovian method:

$$
p_{i}(N, x)=\frac{\lambda_{i}+x_{i}}{\lambda_{N}+x_{N}} \text { for all } N, x \text { and } i
$$

The $\lambda$-fixed chances filling method is:

$$
p_{i}(N, x)=\frac{\lambda_{i}}{\lambda_{N}} \text { for all } N, x \text { and } i
$$


The interpretation of formula (7) is clear. The urn contains balls of different colors, one color per agent. There are $x_{i}$ balls of color $i$ plus a fixed number $\lambda_{i}$ (that may not be an integer) We draw at random a ball from this urn and replace it with two balls of the same color.

The Polya-Eggenberger (in short P.E.) urn-filling methods are a familiar example of discrete stochastic process, and their statistical properties are wellknown: see Chapter 4 in Johnson and Kotz [1977]. Starting from an urn $(N, x)$, the expected value of $r(N, x, t)$ - the urn's content after $t$ balls have been added - is:

$$
E[r(N, x, t)]=x+\frac{t}{\lambda_{N}+x_{N}}(\lambda+x)
$$

where $\lambda$ stands for the projection of $\lambda$ on $\mathbb{R}^{N}$. The random variable $\{r(N, x, t)-$ $x\} / t$ converges in probability to the distribution on the $N$-simplex with density C. $y_{1}^{\lambda_{1}+x_{1}-1} \cdot y_{2}^{\lambda_{2}+x_{2}-1} \ldots y_{n}^{\lambda_{n}+x_{n}-1}$ with respect to the Lebesgue measure $(C$ is a normalization parameter). For instance, with $\lambda_{i}=1$ and $x_{i}=0$ for all $i$, the probability distribution of $r(N, t, x) / t$ converges to the Lebesgue measure on the $N$-simplex.

The $\lambda-P$.E. filling method is share monotonic ${ }_{+}$: increasing agent $i^{\prime} s$ share $x_{i}$ strictly increases the probability $p_{i}(N, x)$; property (5) can be checked with a little more work. The $\lambda$-fixed chances method is both share monotonic $c_{+}$and share monotonic .

Unlike the winner-takes-all methods of Proposition 1, a P.E. or a fixed chances method never leaves out any agent from the distribution process: every agent has a positive probability of receiving the next unit, a property that we call

$$
\text { Positive Shares (PS) : } p_{i}(N, x)>0 \text { for all } N, x \text { and } i
$$

Positive shares is a strong requirement, ruling out for instance all deterministic methods. However, many of the equalizing methods of Proposition 1 meet a weaker property, namely they guarantee that no agent is ever left out if we look far enough in the future:

$$
\begin{aligned}
\text { Positive Future Shares (PFS) } & : \quad \text { for all } N, x \text { and } i, \\
\text { there exists } t \text { such that } E\left[r_{i}(N, x, t)\right] & >x_{i}
\end{aligned}
$$

Positive future shares rules out the possibility that starting from urn $x$, a certain agent $i$ never receives any of the units distributed in the indefinite future. Thus PFS is a mild requirement, that only rules out extreme methods such as the winner-takes-all of Proposition 1.

Theorem 1 Assume $|\mathcal{N}| \geq 3$. The family of the $\lambda$-Polya-Eggenberger (7) and the $\lambda$-fixed chances (8) methods, when $\lambda$ varies over all profiles of positive weights, is characterized by each one of the two following combinations of axioms: 
i) Markovian Property (1), Consistency (2), Share Monotonicity (5) and Positive Future Shares (10);

ii) Markovian Property (1), Consistency (2), and Positive Shares (9);

In Appendix 2, we discuss the family of methods meeting Markovian, Con-

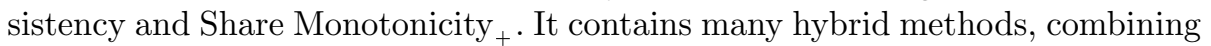
winner-takes-all, P.E. and fixed chances methods.

Checking further that the two characterization results are tight is easy with the help of the two examples (3), (4) in Section 2 and of Proposition 1. We omit the details.

None of the axioms listed in Theorem 1 requires a symmetric treatment of the agents, indeed the methods thus characterized allow for an arbitrary degree of systematic discrimination by adjusting the weights $\lambda$. Consider the basic equity requirement known as:

Equal Treatment of Equals (ETE): for all $N, x, t, i$ and $j$

$$
x_{i}=x_{j} \Rightarrow r_{i}(N, x, t) \text { and } r_{j}(N, x, t) \text { are identically distributed }
$$

Under ETE, the family characterized in Theorem 1 reduces to the (unique) symmetric fixed chances methods $\left(\lambda_{i}=1\right.$, all $\left.i\right)$ and to the one-dimensional family of symmetric PE methods:

$$
p_{i}(N, x)=\frac{\lambda_{0}+x_{i}}{n \cdot \lambda_{0}+x_{N}} \text { for all } N,|N|=n, \text { all } x \text { and } i
$$

where $\lambda_{0}$ is a positive number.

Remark 4 In the urn-emptying model, the counterpart of the Polya-Eggenberger family of methods is a single method called the proportional method and defined as follows. At urn $x$, the probability that the first unit is taken away from agent $i$ is proportional to $x_{i}$. This method is characterized in Moulin [2000b] by several combinations of axioms. One result in particular (statement $i$ in Theorem 3) is the direct counterpart of statement ii in Theorem 1 above: the proportional method is characterized by Consistency, the Markovian property and Positive shares (every agent has a positive probability of losing the next unit; this property is called Positive Risks in Moulin [2000b]).

On the other hand, Positive Future Shares has no counterpart in the urnemptying model, and the combination of Markovian, Consistency and Share Monotonicity ${ }_{+}$is not enough to capture the proportional method alone: more on this combination in Remark 5 at the end of the next section.

The fixed chances methods of the urn-filling model are easy to adapt in the urn-emptying one. Given an urn $x$, only the agents in the support of $x, x_{i}>0$, can lose the next unit; a fixed chances method assigns the probability of losing the next unit among the agents in the support of $x$, in proportion to some fixed weights $\lambda$. However, this method is not consistent, as we show now. 
Consider the (markovian) method giving equal chances of losing the next unit to all agents in the support of $x$. Then for $N=\{1,2,3\}, x=(2,1,1)$ and $t=2$ we have:

$$
\begin{aligned}
\operatorname{proba}\{r(N, x, t) & =(1,1,0)\}=\frac{5}{18} ; \\
\operatorname{proba}\left\{r_{3}(N, x, t)\right. & =0\}=\frac{11}{18} ; \\
\operatorname{proba}\{r(\{1,2\},(2,1), 1) & =(1,1)\}=\frac{1}{2}
\end{aligned}
$$

but $\frac{5}{18} \neq \frac{11}{18} \times \frac{1}{2}$.

\section{$5 \quad$ Equalizing methods}

We investigate the markovian and consistent filling methods satisfying Share Monotonicity_. We already know two subsets of this family, namely the equalizing methods of Proposition 1 and the fixed chances methods (8). Theorem 2 below shows that the general methods combine these two ingredients as well as some restricted proportional methods.

We introduce some notations toward the statement of Theorem 2. Given a -possibly infinite- subset $\mathcal{M}$ of $\mathcal{N}$, a $\mathcal{M}$-interval is a subset $\left[a, b\left[\right.\right.$ of $\mathbb{N}^{\mathcal{M}}$ defined by the inequalities $a_{i} \leq z_{i}<b_{i}$ for $i \in \mathcal{M}$. We assume $a_{i}<b_{i} \leq+\infty$, i.e., $b_{i}$ could be infinite. For any finite subset $M$ of $\mathcal{M}$, we abuse notations by saying that the $M$-vector $z$ is in $\left[a, b\left[\right.\right.$ if $a_{i} \leq z_{i}<b_{i}$ for all $i \in M$.

We say that the $\mathcal{M}$-interval $\left[a, b\left[\right.\right.$ is bounded if $b$ is bounded $\left(b \in \mathbb{N}^{\mathcal{M}}\right)$. We call $|\mathcal{M}|$ the type of our interval. Thus the properties a certain interval is bounded, and its type is finite, are logically independent.

We define next a filling method $\widetilde{r}$ restricted to a given $\mathcal{M}$-interval $[a, b[$. For any finite subset $M$ of $\mathcal{M}$, any $M$-vector $z$ s.t. $a_{i} \leq z_{i} \leq b_{i}$ for all $i \in M$ and $z \neq b$, and any integer $t, 1 \leq t \leq b_{M}-z_{M}$, the method specifies a $M$-random variable $\widetilde{r}(M, z, t)=Y$, where $Y_{i}$ is integer-valued, $z_{i} \leq Y_{i} \leq b_{i}$ for all $i \in M$, and $Y_{M}=z_{M}+t$. Notice that whenever $z_{i}=b_{i}$ (which can only happen if $b_{i}$ is finite) the restricted filling method never gives anymore units to agent $i$.

It is straightforward to extend the definitions of the Markovian property

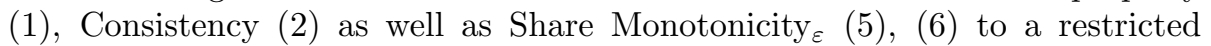
filling method. A markovian method is represented by a probability distribution $\widetilde{p}(M, z)$ defined for all $M$-vectors $z, a \leq z \leq b$ and $z \neq b$, and such that $p_{i}(M, z)>0 \Rightarrow z_{i}<b_{i}$.

An example of interest is the proportional method restricted to a given bounded $\mathcal{M}$-interval $[a, b[$. This is the following markovian method. For all finite subset $M$ of $\mathcal{M}$ and all $M$-vector $z, a \leq z \leq b$ and $z \neq b$ : 


$$
\widetilde{p_{i}}(M, z)=\frac{b_{i}-z_{i}}{b_{M}-z_{M}} \text { for all } i \in M
$$

Note that this method is Share Monotonic_.

Definition 3 A probabilistic standard of comparison (in short, a standard) is a pre-ordering (complete, transitive) $\succsim$ of $\mathcal{N} \times \mathbb{N}$, non-decreasing in the second coordinate: $\left(i, x_{i}\right) \precsim\left(i, x_{i}+1\right)$ for all $i, x_{i}$.

We call $\left(i, x_{i}\right)$ a claim by agent $i$.

An indifference class of the standard $\succsim$ takes the form $\underset{j \in \mathcal{M}}{\cup}\{j\} \times\left[a_{i}, b_{i}[\right.$, where $\mathcal{M}$ is a subset of $\mathcal{N}$ and $a_{i}<b_{i} \leq+\infty$. We shall represent an indifference class of $\succsim$ as the $\mathcal{M}$-interval $[\mathrm{a}, \mathrm{b}[$. We call $|\mathcal{M}|$ the type of the indifference class.

Given an urn $(N, x)$, we write $M(x)$ for the subset of agents $i$ whose claim $\left(i, x_{i}\right)$ is minimal with respect to a given standard $\succsim:$

$$
M(x)=\left\{i \in N \mid\left(i, x_{i}\right) \precsim\left(j, x_{j}\right) \text { for all } j \in N\right\}
$$

The $\mathcal{M}(x)$-interval representing the minimal indifference class of $\succsim$ at $x$ (i.e., the indifference class of $\left(i, x_{i}\right)$ for all $\left.i \in M(x)\right)$ is denoted $I(x)=[a, b[$ (for simplicity we do not make $a, b$ depend explicitly on $x)$.

Note that $M(x)=\mathcal{M}(x) \cap N$. We call $|\mathcal{M}(x)|$ the type of the urn $(N, x)$. If the type of $(x, N)$ is 1 , the standard has a unique smallest claim $\left(i, x_{i}\right)$ at $x$, but the converse is not true: $M(x)$ may be a singleton while $\mathcal{M}(x)$ is not.

Definition 4 Let $\succsim$ be a standard of comparison such that either all indifference classes are bounded, or there is exactly one unbounded class, namely a $\mathcal{N}$-interval $\left[a,+\infty\left[\left(b_{i}=+\infty\right.\right.\right.$ for all $\left.i\right)$. In the latter case, the unbounded class is the highest indifference class of $\succsim$.

$A \succsim$-equalizing urn-filling method is defined by choosing for the unbounded

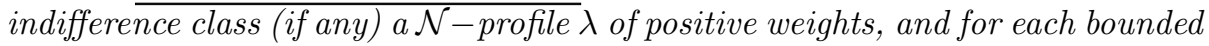
indifference class $[a, b[$ of type 2 (if any), a markovian filling method $\widetilde{p}$ restricted to $[a, b[$.

The $\succsim-e q u a l i z i n g$ method is markovian. For any urn $(N, x)$ not of type 2 the support of $p(N, x)$ is $M(x)$, moreover:

- if the type of $(N, x)$ is at least 3 and $I(x)=[a, b[$ is bounded, our method is the proportional method restricted to $[a, b[$ :

$$
p_{i}(N, x)=\frac{b_{i}-x_{i}}{b_{M(x)}-x_{M(x)}} \text { for all } i \in M(x)
$$


- if the type of $(N, x)$ is at least 3 and $I(x)=[a,+\infty[$ is the unbounded indifference class, then $M(x)=N$ and our method is the $\lambda$-fixed chances:

$$
p_{i}(N, x)=\frac{\lambda_{i}}{\lambda_{N}} \quad \text { all } i \in N
$$

- if the type of $(N, x)$ is two (so $I(x)=[a, b[$ is bounded), our method is the given method $\widetilde{p}$ restricted to $[a, b[$ :

$$
p_{i}(N, x)=\widetilde{p}_{i}\left(M(x), x_{[M(x)]}\right) \quad \text { for all } i \in M(x)
$$

(where $x_{[M]}$ denotes the projection of $x$ on $\mathbb{N}^{M}$ )

The above definition exhausts all cases because if $(N, x)$ is of type $1, M(x)$ reduces to the unique agent $i$ whose claim $\left(i, x_{i}\right)$ is $\succsim-$ minimal.

The last definition before stating Theorem 2 is related to the property Positive Future Shares. We say that a standard $\succsim$ is archimedian if for all claim $\left(i, x_{i}\right)$ and all $j, j \neq i$, there exists a claim $\left(j, x_{j}\right)$ such that $\left(i, x_{i}\right) \precsim\left(j, x_{j}\right)$. This says that any claim by any agent can be reached in the standard by a large enough claim of any other agent.

Note that a standard $\succsim$ with an unbounded $\mathcal{N}$-class $\left[a^{*},+\infty[\right.$, as in Definition 4 , is archimedian.

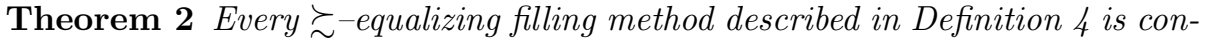
sistent. It meets Positive Future Shares if and only if the standard $\succsim$ is archimedian. It is share monotonic _ if and only if each method $\widetilde{r}$ restricted to a bounded indifference class of type 2 (if any) is share monotonic_.

Conversely, assume $|\mathcal{N}| \geq 3$. A markovian and consistent filling method, satisfying Share Monotonicity_ and Positive Future Shares, is a $\succsim-e q u a l i z i n g$ method as in Definition 4.

The family of methods described in Definition 4 is not simple. A more intuitive subset consists of its equitable members, in the sense of Equal Treatment of Equals (11).

We describe first the symmetric standards of comparison. Observe that any partition of $\mathbb{N}$ in disjoint intervals is described by a finite or infinite strictly increasing sequence in $\mathbb{N} \cup\{+\infty\}, a^{0}=0, a^{1}, \ldots, a^{k}, \ldots$ such that $\sup _{k}\left\{a^{k}\right\}=$ $+\infty$. The partition consists of the intervals $\left[a^{k}, a^{k+1}[\right.$; if it is finite the largest element in the sequence is $+\infty$.

Given such a sequence, we define for all $z \in \mathbb{N}, \tau(z)=k$ iff $a^{k} \leq z<a^{k+1}$, and call $\tau(z)$ the trace of $z$ in this partition.

A standard of comparison $\succsim$ is symmetric if $\left(i, x_{i}\right) \sim\left(j, x_{j}\right)$ whenever $x_{i}=$ $x_{j}$ : two claims of identical sizes are regarded as equivalent by the standard. A symmetric standard is represented by an increasing sequence $a^{0}=0, a^{1}, \ldots, a^{k}, \ldots$ such that $\sup _{k} a^{k}=+\infty$ :

$$
\left(i, x_{i}\right) \succsim\left(j, x_{j}\right) \text { iff } \tau\left(x_{i}\right) \geq \tau\left(x_{j}\right)
$$


and conversely every such sequence defines a symmetric standard. Note that a symmetric standard is archimedian.

Fix a symmetric standard $\succsim$. For any $\operatorname{urn}(N, x)$, we have $M(x)=\arg \min \tau\left(x_{i}\right)$ and $I(x)=\left[a^{k} \mathbf{. 1}, a^{k+1} \mathbf{. 1}\left[\right.\right.$ where $k=\min _{i} \tau\left(x_{i}\right)$ and $\mathbf{1}$ is the $N$-vector with all coordinates equal to $\mathbf{1}$. Note that if $I(x)=\left[a^{k} \mathbf{. 1}, \infty\left[\right.\right.$, we have $\tau\left(x_{i}\right)=k$ for all $i$ and $M(x)=N$.

\section{Corollary to Theorem 2}

Fix a symmetric standard $\succsim$ represented by the sequence $a^{0}=0, a^{1}, \ldots, a^{k}, \ldots$ as above. Consider the following markovian filling method:

$$
\begin{gathered}
\text { for all }(N, x) \text {, let } k=\min _{i} \tau\left(x_{i}\right) \text { and } m(x)=|M(x)| \\
\text { if } a^{k+1}<+\infty: p_{i}(N, x)=\frac{a^{k+1}-x_{i}}{m(x) a^{k+1}-x_{M(x)}} \text { for } i \in M(x) \\
=0 \text { for } \mathrm{i} \notin M(x) \\
\text { if } a^{k+1}=+\infty: p_{i}(N, x)=\frac{1}{n} \text { for all } i
\end{gathered}
$$

This method is consistent, share monotonic_ and satisfies Equal Treatment of Equals (11).

Conversely, assume $|\mathcal{N}| \geq 3$. There are no other markovian, consistent filling methods meeting Share Monotonicity_ and Equal Treatment of Equals.

Each method in the above family is determined by a sequence $a^{0}=0, a^{1}, \ldots, a^{k}$, ...or, equivalently, a partition of $\mathbb{N}$ in intervals. The coarsest partition, $a^{0}=$ $0, a^{1}=+\infty$, yields the equal chances method $\left(p_{i}(N, x)=1 / n\right.$ for all $N, x$ and $i)$. The finest partition, $a^{k}=k, k=0,1,2, \ldots$, yields the method:

$$
p(N, x) \text { is uniformly distributed over argmin } x_{i}
$$

This method is closest to the deterministic equalizing methods of Section 3. Among all methods described in Theorem 2, it deserves best the name equalizing. If two agents have identical shares in urn $(N, x)$ then no matter how many additional units are distributed, the shares of these agents never differ by more than one unit:

$$
x_{i}=x_{j} \Rightarrow\left|r_{j}(N, x, t)-r_{i}(N, x, t)\right| \leq 1 \text { for all } N, x, t, i \text { and } j
$$

One can show that the method (15) is characterized by the combination of Share Monotonicity_ (6), Equal Treatment of Equals (11), property (16), and the Markovian property $(1)^{5}$.

\footnotetext{
${ }^{5}$ The proof is very similar to that of Theorem 1, statement b in Moulin and Stong [2000]. It is omitted for brevity.
} 
Remark 5 The main result (Theorem 2) in Moulin and Stong [2000] characterizes all the markovian, consistent and share monotonic + urn-emptying methods. Their structure is similar to that of the filling methods in Theorem 2 above. There is a standard of comparison $\succsim$ and at urn $x$ the agent who loses the next unit is among those with the smallest claim $\left(i, x_{i}\right)$. Within an indifference class of type at least three, a restricted proportional method is used. The only difference is that the fixed chances methods play no role any more (recall from Remark 4 that such a method is not consistent in the urn-emptying model).

Not surprisingly, the proof technique of our Theorem 2 follows closely the proof of that result.

\section{Invariance under Transfers and Order Inde- pendence}

We introduce a powerful independence property that both the fixed chances and the Polya-Eggenberger methods satisfy. In turn this leads to a new characterization of (a variant of) this family.

Independence of Transfers is one of the oldest ideas in the axiomatic literature on fair division according to claims (Banker [1981], Moulin [1987], De Frutos [1999]): it rules out the profitability of interpersonal transfers of shares.

Invariance under Transfers (IT): for all $N, x, x^{\prime}, t$ and all $S, S \subseteq N$

$$
\begin{aligned}
\left\{x_{S}=\right. & \left.x_{S}^{\prime} ; x_{i}=x_{i}^{\prime} \text { for all } i \notin S\right\} \Rightarrow \\
& \left\{r_{S}(N, x, t) \text { and } r_{S}\left(N, x^{\prime}, t\right) \text { are identically distributed }\right\}
\end{aligned}
$$

The IT axiom rules out the following kind of strategic maneuver. A coalition $S$ of agents transfers some of the shares of its members, thus transforming the initial profile $\left(x_{i}, i \in S\right)$ to $\left(x_{i}^{\prime}, i \in S\right)$; feasibility of these transfers requires $x_{S}=x_{S}^{\prime}$. When $t$ units are distributed, the total share accruing to coalition $S$ may be affected by the transfers: in this case, these agents can manipulate the method to their mutual advantage by a combination of transferring shares twice, before and after the filling method is applied.

Under IT, individual shares are like anonymous bonds that can be passed around between players without affecting their "returns". An important consequence of property (17) is that the probability distribution of agent $i$ 's share $Y_{i}$ only depends upon his initial share $x_{i}$ and the aggregate share of the agents in $N \backslash i: r_{i}(N, x, t)$ takes the form $\rho_{i}\left(N, x_{i}, x_{N}, t\right)^{6}$.

Clearly the fixed chances and P.E. methods in Definition 2 meet IT. On the other hand, a winner-takes-all or an equalizing method in Definition 1 violates this property. The same is true of most equalizing methods in Definition 4: for

\footnotetext{
${ }^{6}$ This follows from applying (17) to $S=N \backslash i$. The converse property is just as easy: if the distribution of $Y_{i}$ only depends upon $x_{i}$ and $x_{N}$, then IT holds.
} 
instance, from the family described in the Corollary to Theorem 2, only the fixed chances methods pass the IT test.

Our last axiom expresses that the distribution of successive units in the urn is equally likely in any ordering of the recipients.

Given a markovian filling method $r$ and an urn $(N, x)$, the successive allocation of $t$ units generates a random sequence in $N, \sigma=\left\{i_{1}, i_{2}, \ldots, i_{t}\right\}$, where $i_{k}$ is the agent receiving the $k-t h$ unit.

We say that two sequences $\sigma=\left\{i_{1}, \ldots, i_{t}\right\}$ and $\sigma^{\prime}=\left\{j_{1}, \ldots, j_{t}\right\}$ in $N$ are permutations of one another if we can write $j_{k}=i_{p(k)}$ for $k=1, \ldots, t$, where $p$ is a bijection from $\{1, \ldots, t\}$ into itself.

Order Independence $(O I)$ : for all $N, x$ and $t$

$$
\begin{aligned}
\sigma^{\prime} & =\left\{j_{1}, \ldots, j_{t}\right\} \text { is a permutation of } \sigma=\left\{i_{1}, \ldots, i_{t}\right\} \\
& \Rightarrow\left\{\sigma \text { and } \sigma^{\prime} \text { are equally probable under } r \text { at }(N, x)\right\}
\end{aligned}
$$

If $p(N, x)$ is the probability distribution of the first unit, the $O I$ property is:

$$
p_{i_{1}}(x) \cdot p_{i_{2}}\left(x+e_{i_{1}}\right) \ldots . . p_{i_{t}}\left(x+\sum_{1}^{t-1} e_{i_{k}}\right)=p_{j_{1}}(x) \cdot p_{j_{2}}\left(x+e_{j_{1}}\right) \ldots . . p_{j_{t}}\left(x+\sum_{1}^{t-1} e_{j_{k}}\right)
$$

whenever $\sigma$ and $\sigma^{\prime}$ are permutations of one another.

Order Independence does not place any direct restriction on the aggregate distribution of $t$ units, namely on the distribution of the random variable $r(N, x, t)$. For instance, it is satisfied by a "dictatorial" method allocating all units to a certain agent $i^{*}$ (the dictator) irrespective of the urn $(N, x)$ : all sequences $\sigma$ generated by this method are constant at $i_{t}=i^{*}$. The winnertakes-all methods of Proposition 1 meet OI for the same reason.

Order Independence is a neutrality property, stating that no agent is systematically served earlier than another agent when units are distributed sequentially. If -say-, $N=\{1,2\}$ and agent 1 must receive on average twice as many units than agent 2 when one hundred units are distributed, then he is equally likely to receive all his units first or all units last, or in any other timing.

Note that OI does not make sense for a non-markovian filling method $r$, because the independent draws of $e_{i_{1}}, e_{i_{2}}, \ldots$ do not sum to a (random) urn $Y=x+\sum_{1}^{t} e_{k}$ with the same distribution as $r(N, x, t)$.

A fixed chances method (8) satisfies Order Independence: this is obvious on equation (18). A P.E. method meets OI as well, as shown by developing both sides of (18) with the help of (7). On the other hand, the equalizing methods of Definitions 1 and 4 fail OI.

The three axioms M, IT and OI involve a fixed population: they do not impose any relation between the methods to fill urns $(N, x)$ and $\left(N^{\prime}, x^{\prime}\right)$ when 
$N$ and $N^{\prime}$ are two different "populations". Therefore we fix a finite population $N$ in our next result.

Theorem 3 Fix the finite set $N$ of concerned agents and assume $|N| \geq 3$. To a profile of non-negative weights $\lambda_{i}, i \in N$, not all zero, we associate three (fixed population) markovian urn-filling methods:

i) fixed chances: for all $x, i$

$$
p_{i}(N, x)=\frac{\lambda_{i}}{\lambda_{N}}
$$

ii) quasi-proportional: for all $x, i$

$$
p_{i}(N, x)=\frac{x_{i}}{x_{N}} \text { if } x_{N}>0, p_{i}(N, 0)=\frac{\lambda_{i}}{\lambda_{N}}
$$

iii) Polya-Eggenberger:

$$
p_{i}(N, x)=\frac{\lambda_{i}+x_{i}}{\lambda_{N}+x_{N}} \text { for all } x, i
$$

These methods are independent of transfers and order independent. Conversely, there is no other markovian method meeting IT and OI.

In Appendices 6 and 5, we discuss two families of methods characterized respectively by the 3 axioms M, CSY, IT, or by the triple M, CSY, OI. We

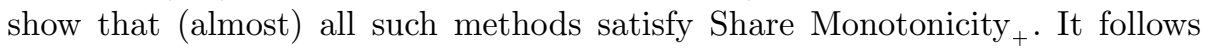
that if we add PFS to the list of requirements, we are back in each case to the fixed chances and P.E. methods of Theorem 1. On the other hand, when PFS is not required, the combination M, CSY, IT captures new methods mixing fixed chances, P.E. and proportional in interesting ways. By contrast, the triple M, CSY, OI has essentially the same bite as M, CSY, $\mathrm{SM}_{+}$discussed in Appendix 2.

Remark 6 In the urn-emptying model, the proportional method meets Independence of Transfers and Order Independence. In fact, OI is essentially a rewriting of the definition of this method and IT is enough to single out this method among all markovian methods ${ }^{7}$. Thus either one of the two axioms IT and OT leaves no choice whatsoever to the mechanism designer in the urn-emptying case.

${ }^{7}$ This result is not to be found in the two companion papers, that discuss neither IT nor OI. It is an easy consequence of Step 1 the proof of Theorem 3 in Appendix 3. 


\section{Bayesian filling methods}

Now let us consider the methods that satisfy just $M$ and OI. Since M and OI do not make use of a variable population, we may assume that there is a fixed population $N$. Let $\Sigma(N)=\left\{\left(p_{i}\right)_{i \in N}: 0 \leq p_{i} \leq 1\right.$, and $\sum_{i \in N} p_{i}=$ $1\}$ be the $|N|-1$ dimensional simplex and let $\stackrel{\circ}{\Sigma}(N)$ denote its interior. We may think of a point in $\Sigma(N)$ as an assignment of a probability to each of the agents. A Bayesian assignment method is determined by the choice of a probability measure $\pi$ on Borel subsets of $\Sigma(N)$, with the technical restriction that $\pi(\stackrel{\circ}{\Sigma}(N))>0$.

Given such a measure, we can describe the Bayesian method in either of two equivalent fashions. For any vector $a=\left(a_{i}\right)_{i \in N}$ of non negative integers, define the $a$ th moment to be $M(a)=\int_{\Sigma(N)} \prod_{i \in N} p_{i}^{a_{i}} d \pi(p)$. Note that because of our technical assumption, $M(a)>0$ for all $a$. Then we can define a markovian urn-filling method by the formula $p_{i}(N, x)=M\left(x+e_{i}\right) / M(x)$.

Alternately, define measures $\pi_{x}$ by

$$
\pi_{x}(A)=\frac{\int_{A} \prod_{i \in N} p_{i}^{x_{i}} d \pi(p)}{\int_{\Sigma(N)} \prod_{i \in N} p_{i}^{x_{i}} d \pi(p)} .
$$

Given initial claims of $x$, we choose a set of probabilities $\left(p_{i}\right)_{i \in N}$ randomly from $\Sigma(N)$ according to the probability measure $\pi_{x}$ and then we color successive balls independently at random according to the chosen probabilities. In Appendix 3 we will show that this is equivalent to the description above.

With these definitions we have the following theorem.

Theorem 4 An urn-filling method that meets the Markovian property, Order Independence and Positive Future Shares is a bayesian method. Conversely, any bayesian method meets the Markovian property, Order Independence, Positive Future Shares, Positive Shares, and Share Monotonicity+.

In Appendix 4, we give the proof of this Theorem and in the process also give a rather complicated characterization of the family of methods satisfying just the Markovian Property and Order Independence. 


\section{APPENDIX 1: PROPOSITION 1 AND THEOREM 1}

\section{Proposition 1}

The simple proof is similar to that of Proposition 1 in Moulin and Stong [2000]. The "if" statements are clear.

Let $r$ be a markovian and consistent deterministic method meeting $S M_{+}^{1}$ (Remark 1 ). We write $\pi(N, x)=i$ whenever $p(N, x)$ chooses $i$ with probability 1 . We write $\pi(x)$ for simplicity when the set of coordinates of the profile $x$ is clear. For instance $\pi\left(x_{i} e_{i}+x_{j} e_{j}\right)$ stands for $\pi\left(\{i, j\}, x_{i} e_{i}+x_{j} e_{j}\right)$. Define a binary relation $\succ$ on $\mathcal{N} \times \mathbb{N}$ :

$$
\left(i, x_{i}\right) \succ\left(j, x_{j}\right) \quad \text { iff }\left\{i=j \text { and } x_{i}>x_{j}\right\} \text { or }\left\{i \neq j \text { and } \pi\left(x_{i} e_{i}+x_{j} e_{j}\right)=i\right\}
$$

Clearly $\succ$ is complete and antisymmetric. It is transitive as well. For instance assume $i, j, k$ are all different and

$$
\pi\left(x_{i} e_{i}+x_{j} e_{j}\right)=i ; \pi\left(x_{j} e_{j}+x_{k} e_{k}\right)=j
$$

Consistency implies:

$$
\begin{aligned}
& \pi\left(x_{i} e_{i}+x_{j} e_{j}+x_{k} e_{k}\right)=j \Rightarrow \pi\left(x_{i} e_{i}+x_{j} e_{j}\right)=j \\
& \pi\left(x_{i} e_{i}+x_{j} e_{j}+x_{k} e_{k}\right)=k \Rightarrow \pi\left(x_{j} e_{j}+x_{k} e_{k}\right)=k
\end{aligned}
$$

Therefore $\pi\left(x_{i} e_{i}+x_{j} e_{j}+x_{k} e_{k}\right)=i$, implying $\pi\left(x_{i} e_{i}+x_{k} e_{k}\right)=i$, as desired. Checking transitivity when $i, j, k$ are not all different, is easy with $S M_{+}^{1}$.

Repeatedly applying CSY at an arbitrary urn $(N, x)$, we get:

$$
\pi(N, x)=i \Rightarrow \pi\left(N \backslash k, x_{-k}\right)=i \Rightarrow \ldots \Rightarrow \pi\left(x_{i} e_{i}+x_{j} e_{j}\right)=i
$$

for any pair $i, j$ in $N$. Thus $\pi(N, x)=i$ implies $\left(i, x_{i}\right) \succ\left(j, x_{j}\right)$ for all $j$ in $N \backslash i$, and $r$ is the $\succ-$ winner-takes-all method of Definition 1 as desired. Turning to the case of a markovian and consistent method meeting $S M_{-}^{1}$, we define the relation $\succ$ as follows:

$$
\left(i, x_{i}\right) \succ\left(j, x_{j}\right) \text { iff }\left\{i=j \text { and } x_{i}>x_{j}\right\} \text { or }\left\{i \neq j \text { and } \pi\left(x_{i} e_{i}+x_{j} e_{j}\right)=j\right\}
$$

One checks as above that $\succ$ is a linear ordering of $\mathcal{N} \times \mathbb{N}$. The only difference is when checking transitivity in cases where $i, j, k$ are not all distinct, e.g.:

$$
\left(i, x_{i}\right) \succ\left(i, x_{i}^{\prime}\right) \text { and }\left(i, x_{i}^{\prime}\right) \succ\left(j, x_{j}\right)
$$


Here $x_{i}>x_{i}^{\prime}$ and $p_{i}\left(x_{i}^{\prime} e_{i}+x_{j} e_{j}\right)=0$, so $S M_{-}^{1}$ implies $p_{i}\left(x_{i} e_{i}+x_{j} e_{j}\right)=0$ as desired.

Property (19) implies that $r$ is the $\succ$-equalizing method of Definition 1.

\section{Theorem 1}

Statement ii

We fix $r$, a P.E method (7), and check that it is consistent. For any positive integer $a$ and non-negative integer $b$, we use the notation $(a, b) !=a \cdot(a+1) \cdot(a+$ $2) \ldots .(a+b-1)$ with the convention $(a, 0) !=1$.

Fix $N, x, t, y$ and consider a sequence $\sigma$ in $N$ where $j$ appears $z_{j}=y_{j}-$ $x_{j}$ times, for all $j$. The probability that $\sigma$ is the realization of $t$ independent successive allocations of one unit according to (7) is:

$$
\operatorname{proba}(\sigma)=\frac{\left(\lambda_{1}+x_{1}, z_{1}\right) ! \ldots\left(\lambda_{n}+x_{n}, z_{n}\right) !}{\left(\lambda_{N}+x_{N}, t\right) !}
$$

Because $r$ is markovian, the probability distribution of $r(N, t, x)$ obtains from $t$ successive independent allocations of one unit, therefore:

$$
\operatorname{proba}\{r(N, x, t)=y\}=\frac{t !}{z_{1} ! \ldots . z_{n} !} \cdot \frac{\left(\lambda_{1}+x_{1}, z_{1}\right) ! \ldots\left(\lambda_{n}+x_{n}, z_{n}\right) !}{\left(\lambda_{N}+x_{N}, t\right) !}
$$

similarly

$$
\operatorname{proba}\left\{r_{i}(N, x, t)=y_{i}\right\}=\frac{t !}{z_{i} ! z_{N \backslash i} !} \cdot \frac{\left(\lambda_{1}+x_{1}, z_{1}\right) !\left(\lambda_{N \backslash 1}+x_{N \backslash 1}, z_{N \backslash 1}\right) !}{\left(\lambda_{N}+x_{N}, t\right) !}
$$

and equality (2) follows easily.

For a fixed chances method (8), Consistency can be checked in similar fashion. Alternatively, we can use the fact that a fixed chances method is the limit of a sequence of P.E. methods where the $\lambda_{i}$ all tend to infinity at the same rate. Since the CSY equation is a rational function of the $\lambda_{i}$, it follows that the limit method is consistent.

Thus all P.E. and fixed chances methods are consistent, and they obviously meet PS.

We turn to the converse statement. Fix a markovian and consistent method $r$ meeting PS. Consistency (2) applied to $t=1$ gives:

$$
p_{i}(N, x)=\left(1-p_{j}(N, x)\right) \cdot p_{i}\left(N \backslash j, x_{-j}\right) \quad \text { for all } N, x, i, j
$$

PS implies $0<p_{k}(N, x)<1$ for all $N, x, k$. Applying (20) repeatedly:

$$
\frac{p_{i}(N, x)}{p_{j}(N, x)}=\frac{p_{i}\left(x_{i} e_{i}+x_{j} e_{j}\right)}{p_{j}\left(x_{i} e_{i}+x_{j} e_{j}\right)} \quad \text { for all } N, x, i, j
$$


(where $p_{i}\left(x_{i} e_{i}+x_{j} e_{j}\right)$ stands, as usual, for $p_{i}\left(\{i, j\}, x_{i} e_{i}+x_{j} e_{j}\right)$ ).

By assumption $\mathcal{N}$ contains three distinct agents $1,2,3$. Set $N=\{1,2,3\}$, choose $x$ in $\mathbb{N}^{N}$ and compute:

$$
\frac{p_{1}}{p_{2}}\left(x_{1} e_{1}+x_{2} e_{2}\right) \cdot \frac{p_{2}}{p_{3}}\left(x_{2} e_{2}+x_{3} e_{3}\right) \cdot \frac{p_{3}}{p_{1}}\left(x_{1} e_{1}+x_{3} e_{3}\right)=\frac{p_{1}}{p_{2}}(N, x) \cdot \frac{p_{2}}{p_{3}}(N, x) \cdot \frac{p_{3}}{p_{1}}(N, x)=1
$$

Fixing $x_{3}$, say $x_{3}=0$, the above equation shows the existence of two positive functions $f_{1}\left(x_{1}\right), f_{2}\left(x_{2}\right)$ such that:

$$
\frac{p_{1}}{p_{2}}\left(x_{1} e_{1}+x_{2} e_{2}\right)=\frac{f_{1}\left(x_{1}\right)}{f_{2}\left(x_{2}\right)} \Leftrightarrow p_{i}\left(x_{1} e_{1}+x_{2} e_{2}\right)=\frac{f_{i}\left(x_{i}\right)}{f_{1}\left(x_{1}\right)+f_{2}\left(x_{2}\right)} \quad \text { for } i=1,2 \text {. }
$$

Repeating the argument for all pairs $\{i, j\}$ from $\{1,2,3\}$, and then for all triple $\{i, j, k\}$ in $\mathcal{N}$, we conclude that there exists for all $i \in \mathcal{N}$ a positive function $f_{i}$ such that:

$$
p_{i}\left(x_{i} e_{i}+x_{j} e_{j}\right)=\frac{f_{i}\left(x_{i}\right)}{f_{i}\left(x_{i}\right)+f_{j}\left(x_{j}\right)} \text { for all } i, j, x_{i}, x_{j}
$$

In view of (21) this equally extends to:

$$
p_{i}(N, x)=\frac{f_{i}\left(x_{i}\right)}{\sum_{N} f_{j}\left(x_{j}\right)} \text { for all } N, x, i
$$

The last step of the proof is to show that all functions $f_{i}$ are affine and have identical slopes. For this we must apply CSY (2) to the allocation of at least two units $^{8}$. Fix $N=\{1,2,3\}$ and $x=\left(x_{1}, x_{2}, x_{3}\right)$, and invoke CSY:

$$
\operatorname{proba}\{r(N, x, 2)=(1,1,0)\}=\operatorname{proba}\left\{r_{2}(N, x, 2)=1\right\} \cdot p_{1}\left(x_{1} e_{1}+x_{3} e_{3}\right)
$$

We develop these two probabilities with (22) and the Markovian property. For simplicity, we write $a_{i}=f_{i}\left(x_{i}\right)$ and $b_{i}=f_{i}\left(x_{i}+1\right)$ :

$$
\begin{aligned}
& \operatorname{proba}\{r(N, x, 2)=(1,1,0)\}=\frac{a_{1}}{a_{123}} \cdot \frac{a_{2}}{b_{1}+a_{23}}+\frac{a_{2}}{a_{123}} \cdot \frac{a_{1}}{b_{2}+a_{13}} \\
& \operatorname{proba}\left\{r_{2}(N, x, 2)=1\right\}=\frac{a_{1}}{a_{123}} \cdot \frac{a_{2}}{b_{1}+a_{23}}+\frac{a_{2}}{a_{123}} \cdot \frac{a_{13}}{b_{2}+a_{13}}+\frac{a_{3}}{a_{123}} \cdot \frac{a_{2}}{b_{3}+a_{12}}
\end{aligned}
$$

Developing (23) gives now $b_{1}-a_{1}=b_{3}-a_{3}$, namely $f_{1}\left(x_{1}+1\right)-f_{1}\left(x_{1}\right)=$ $f_{3}\left(x_{3}+1\right)-f_{3}\left(x_{3}\right)$. As the agents 1,3 and the shares $x_{1}, x_{3}$ are arbitrary, we conclude that all functions $f_{i}$ are affine and have the same slope. Because

\footnotetext{
${ }^{8}$ The following proof is similar to that of Theorem 3 in Moulin [2000b].
} 
$f_{i}\left(x_{i}\right)>0$, this leaves only two cases. If the $f_{i}$ are all constant, we have a fixed chance method; if they are all increasing at the same pace, we have a P.E. method.

\section{$\underline{\text { Statement i }}$}

Choose a markovian and consistent method $r$ meeting $S M_{+}^{1}$ and PFS. We prove below that it satisfies PS as well, so the conclusion follows from the statement ii just proved.

Suppose $p_{1}(N, x)=1$ for some $N, x$. Then by $S M_{+}^{1}$ and the Markovian property, the urn $(N, x)$ will be filled exclusively for agent 1 , for any $t$, which contradicts PFS. Thus $p_{1}(N, x)<1$.

Suppose now $p_{1}(N, x)=0$ for some $N, x$. By (20) and the fact that $p$ is never deterministic, $p_{1}\left(N^{\prime}, x^{\prime}\right)=0$ holds for any subset $N^{\prime}$ of $N$ containing 1 , if $x^{\prime}$ is the projection of $x$, and for any superset $N^{\prime}$ of $N$ if $x$ is the projection of $x^{\prime}$. Thus we can assume $N=\{1,2,3\}$. From (20) again we have:

$$
p_{1}\left(x_{1} e_{1}+x_{3} e_{3}\right)=0 \Rightarrow p_{1}\left(N, x+e_{2}\right)=0
$$

And by induction $p_{1}\left(N, x^{\prime}\right)=0$ for any $x^{\prime}$ such that $x_{1}^{\prime}=x_{1}$, and $x_{i}^{\prime} \geq x_{i}$ for $i=2,3$. But this contradicts PFS because the urn $(N, x)$ will be never be filled with any ball of color 1 .

\section{APPENDIX 2: PROOF OF THEOREM 2 AND A GENERALIZATION OF THEOREM 1}

\section{Theorem 2}

Step 1. An equalizing method is consistent

We fix a $\succsim-$-equalizing method $r$ as in Definition 4 . We also fix an urn $\left(N, x^{0}\right)$. We construct first an increasing sequence $x^{0}, x^{1}, \ldots, x^{k}, \ldots$ corresponding to the successive indifference classes $I(y)$, as $y$ varies in the support of $r(N, x, t)$. The important fact is that the sequence is deterministic: for any $t$, all realizations of $Y=r(N, x, t)$ lead to the same class $I(y)$.

Given any urn $(N, x)$ we define a vector $s(x)$ in $\mathbb{N}^{N}$ as follows:

$$
\begin{aligned}
& s(x)_{i}=b_{i} \quad \text { if } i \in M(x) \text { and } I(x)=[a, b[ \\
& s(x)_{j}=x_{j} \quad \text { if } j \notin M(x)
\end{aligned}
$$

Note that $s(x) \geq x, s(x) \neq x$. By definition 4 if $t$ is in the interval $1 \leq t \leq$ $s_{N}(x)-x_{N}$, the support of $r(N, x, t)$ remains in $[x, s(x)]$ and for any $y$ in this support, with the exception of $s(x), I(y)=I(x)$ and $s(y)=s(x)$. Note that $s(x)$ is unbounded only if $I(x)$ is the unbounded indifference class $[a,+\infty[$ of Definition 4. 
We define inductively the sequence $x^{k}$, by $x^{k+1}=s\left(x^{k}\right)$. The successive indifference classes $I\left(x^{k}\right)$ are increasing. If one such class is $\left[a^{*},+\infty[\right.$, the sequence stops there, otherwise it is unbounded. We let $\widetilde{r}^{k}$ to be the filling method restricted to $I\left(x^{k}\right)$ as in Definition 4. Note that each method $\widetilde{r}^{k}$ is consistent: this is clear for the proportional and fixed chances method, and for a method restricted to an interval $[a, b[$ of type 2 , Consistency is vacuously true. This is all we need to show the Consistency of $r$.

Set $M^{k}=M\left(x^{k}\right)$ and $t^{k}=x_{N}^{k}-x_{N}^{0}$, so the sequence $t^{k}$ is strictly increasing and $\sup _{k} t^{k}=+\infty$, whether the sequence $x^{k}$ is finite or infinite. Now $r\left(N, t, x^{0}\right)$ is computed as follows:

$$
\begin{array}{rlrl}
\text { let } k \text { be defined by } t^{k} & <t \leq t^{k+1}: & \\
r_{i}\left(N, x^{0}, t\right) & ={\widetilde{r_{i}}}^{k}\left(M^{k}, x_{\left[M^{k}\right]}^{k}, t-t^{k}\right) & & \text { if } i \in M^{k} \\
& =x_{i}^{k}=x_{i}^{k+1} & & \text { if } i \notin M^{k}
\end{array}
$$

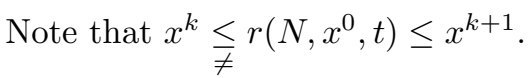

We claim that the sequence $\left(x_{-i}\right)^{k}, k=1,2 \ldots$, for the urn $\left(N \backslash i, x_{-i}^{o}\right)$ is simply $\left(x^{k}\right)_{-i}, k=1,2$, the projection of $x^{k}$ on $N \backslash i$. Indeed, $s\left(x_{-i}\right)=(s(x))_{-i}$ whenever $M(x) \neq\{i\}$, and $s\left(x_{-i}\right)=x_{-i}$ when $M(x)=\{i\}$. Therefore the claim is true, provided we allow a repetition, $x_{-i}^{k}=x_{-i}^{k+1}$, every time $M\left(x^{k}\right)=\{i\}$. We set $t_{(-i)}^{k}=x_{N \backslash i}^{k}-x_{N \backslash i}^{0}$.

We are now ready to prove CSY, namely equation (2), where we fix $t, t^{k}<$ $t \leq t^{k+1}$, an agent 1 , and a profile $y$ in the support of $r\left(N, x^{o}, t\right)$, namely $x^{k} \underset{\neq}{ } \leq x^{k+1}$ and $y_{N}=t$ (if $y$ is outside this support, both sides of (2) are obviously zero).

We distinguish two cases. First we assume $1 \notin M^{k}$. In this case $y^{1}=x_{1}^{k}=$ $x_{1}^{k+1}$, implying proba $\left\{r_{1}(N, x, t)=y_{1}\right\}=1$. Moreover:

$$
t_{(-1)}^{k}<t^{k}-y_{1}+x_{1}^{0}<t-y_{1}+x_{1}^{0} \leq t^{k+1}-y_{1}+x_{1}^{0}=t_{(-1)}^{k+1}
$$

Formula (24) gives:

$$
\operatorname{proba}\left\{r\left(N, x^{0}, t\right)=y\right\}=\operatorname{proba}\left\{\widetilde{r}^{k}\left(M^{k}, x_{\left[M^{k}\right]}^{k}, t-t^{k}\right)=y_{\left[M^{k}\right]}\right\}
$$

similarly, as $M\left(x_{-1}^{k}\right)=M^{k},(24)$ gives

$$
\begin{aligned}
\operatorname{proba}\left\{r\left(N \backslash 1, x_{-1}^{0}, t-y_{1}+x_{1}\right)\right. & \left.=y_{-1}\right\}= \\
\operatorname{proba}\left\{\widetilde{r}^{k}\left(M^{k}, x_{\left[M^{k}\right]}^{k}, t-y_{1}+x_{1}^{0}-t_{(-1)}^{k}\right)\right. & \left.=y_{\left[M^{k}\right]}\right\}
\end{aligned}
$$

The desired quality follows from $y_{1}=x_{1}^{k} \Rightarrow y_{1}-x_{1}^{0}+t_{(-1)}^{k}=t^{k}$. 
The second case is when $M^{k}$ contains 1 , so that $x_{1}^{k}<x_{1}^{k+1}$. We check first:

$$
t_{(-1)}^{k} \leq t-y_{1}+x_{1}^{0} \leq t_{(-1)}^{k+1}
$$

Note that $t-t^{k}=y_{M^{k}}-x_{M^{k}}^{k}$, because $y_{j}=x_{j}^{k}$ for all $j \notin M^{k}$ (see (24)).

Moreover $x^{k} \leq y \leq x^{k+1}$. Using the notation $\widetilde{M}^{k}=M^{k} \backslash 1$ we compute:

$$
\begin{aligned}
t & =y_{M^{k}}-x_{M^{k}}^{k}+x_{N}^{k}-x_{N}^{0}=\left(y_{\widetilde{M}^{k}}-x_{\widetilde{M}^{k}}^{k}\right)+\left(y_{1}-x_{1}^{0}\right)+t_{(-1)}^{k} \\
& \Rightarrow y_{1}-x_{1}^{0}+t_{(-1)}^{k} \leq t \leq x_{\widetilde{M}^{k+1}}^{k+1}-x_{\widetilde{M}^{k}}^{k}+y_{1}-x_{1}^{0}+t_{(-1)}^{k}=t_{(-1)}^{k+1}+y_{1}-x_{1}^{0}
\end{aligned}
$$

implying (25).

By Consistency of $\widetilde{r}^{k}$, we have:

$$
\begin{aligned}
\operatorname{proba}\left\{\widetilde{r}^{k}\left(M^{k}, x_{\left[M^{k}\right]}^{k}, t-t^{k}\right)\right. & \left.=y_{\left[M^{k}\right]}\right\}= \\
\operatorname{proba}\left\{\widetilde{r}_{1}^{k}\left(M^{k}, x_{\left[M^{k}\right]}^{k}, t-t^{k}\right)\right. & \left.=y_{1}\right\} . \\
\operatorname{proba}\left\{\widetilde{r}^{k}\left(M^{k} \backslash 1, x_{\left[\widetilde{M}^{k}\right]}^{k}, t-t^{k}-y_{1}-x_{1}^{k}\right)\right. & \left.=y_{\left[\widetilde{M}^{k}\right]}\right\}
\end{aligned}
$$

By (24), the left-hand and the middle probabilities in the above equality are, respectively, $\operatorname{proba}\left\{r\left(N, x^{0}, t\right)=y\right\}$ and $\operatorname{proba}\left\{r_{1}\left(N, x^{0}, t\right)=y_{1}\right\}$. The righthand probability is $\operatorname{proba}\left\{r\left(N \backslash 1, x_{-1}, t-y_{1}+x_{1}^{0}\right)=y_{-1}\right\}$ by virtue of inequalities (25), of (24) applied to $N \backslash 1$, and of the identity $\left(t-y_{1}+x_{1}^{0}\right)-t_{(-1)}^{k}=$ $t-t^{k}-y_{1}+x_{1}^{k}$.

\section{Step 2}

We check that a method in Definition 4 meets Positive Future Shares if and only if $\succsim$ is archimedian. Assume $\succsim$ is archimedian. Suppose (10) fails at some $N, x, i$, namely $r_{i}(N, x, t)=x_{i}$ with probability 1 for all $t$. In terms of the sequence starting from $x$ constructed in Step 1, this means $i \notin M^{k}=M\left(x^{k}\right)$ for all $k=1,2, \ldots$ If the sequence $x^{k}$ is finite, at some point $I\left(x^{k}\right)=\left[a^{*},+\infty[\right.$ and $M^{k}=N$ (because this is the largest indifference class, see Definition 4), contradiction. If $x^{k}$ is infinite, there exists an agent $j$ and a subsequence $k^{\prime}$ of $k$ such that $x_{j}^{k^{\prime}}$ is unbounded and $j \in M^{k^{\prime}}$ for all $k^{\prime}$. By the archimedian property, $\left(j, x_{j}^{k}\right) \succsim\left(i, x_{i}\right)$ for $k$ large enough, contradicting $i \notin M^{k}$.

Conversely, if $r$ meets PFS, for all $N, x, i$ there exists a term $x^{k}$ in the sequence starting from $x$ such that $i \in M^{k}$. If we take the first such $k$, we have $\left(j, x_{j}^{k}\right) \succsim\left(i, x_{i}\right)$ for all $j$.

If a method of Definition 4 is Share Monotonic , all the restricted methods $\widetilde{r}$ must meet $\mathrm{SM}_{-}$as well. The argument is straightforward.

Conversely, let $r$ be a method as in Definition 4 such that all restricted methods $\widetilde{r}$ meet $S M_{-}$. To check that $r$ is $S M_{-}$, we must compare the two sequences $x^{k}$ and $z^{k}$ generated respectively by $r(N, x, \cdot)$ and $r\left(N, x+e_{i}, \cdot\right)$ : see 
the construction of Step 1. Denote $M^{k}=M\left(x^{k}\right)$ and $P^{k}=M\left(z^{k}\right)$ and let $k^{*}$ be the smallest integer such that $i \in M^{k}$. Then:

$$
\begin{array}{rlrl}
i & \notin P^{k}=M^{k} \text { and } z^{k}=x^{k}+1 \quad \text { for } k<k^{*} \\
P^{k} & =M^{k} \quad \text { and } z^{k}=x^{k} \quad \text { for } k^{*}<k \\
P^{k^{*}} & =M^{k^{*}} \backslash i \text { if } x_{i}^{k^{*}+1}-x_{i}^{k^{*}}=1 & \\
P^{k^{*}} & =M^{k^{*}} \quad \text { if } x_{i}^{k^{*}+1}-x_{i}^{k^{*}}>1 &
\end{array}
$$

From this we deduce with the notations of Step 1, in particular $t^{k}=x_{N}^{k}-$ $x_{N}$, and the symbol $\equiv$ for the fact that two random variables have identical distributions:

$$
\begin{aligned}
r_{i}\left(N, x+e_{i}, t\right) & \equiv r_{i}(N, x, t)+1 \text { for } 1 \leq t \leq t^{k^{*}} \\
r_{i}\left(N, x+e_{i}, t-1\right) & \equiv r_{i}(N, x, t) \text { for } t^{k^{*}+1}<t \\
r_{i}\left(N, x+e_{i}, t\right) & =x_{i}+1 \leq r_{i}(N, x, t)+1 \text { for } t^{k^{*}}<t \leq t^{k^{*}+1}, \text { if } x_{i}^{k^{*}+1}-x_{i}^{k^{*}}=1
\end{aligned}
$$

The inequality (6) follows in each case. The only case left is $t^{k^{*}}<t \leq t^{k^{*}+1}$ when $x_{i}^{k^{*}+1}-x_{i}^{k^{*}}>1$, so that $P^{k^{*}}=M^{k^{*}}$. In this case $r_{i}\left(N, x+e_{i}, t\right)$ and $r_{i}(N, x, t)$ are given by the same restricted method $\widetilde{r}^{k^{*}}$ as in (24):

$$
\begin{aligned}
r_{i}\left(N, x+e_{i}, t\right) & =\widetilde{r}_{i}^{k^{*}}\left(M_{k^{*}}, x_{\left[M^{\left.k^{*}\right]}\right.}^{k^{*}}+e_{i}, t-t^{k^{*}}\right) \\
r_{i}(N, x, t) & =\widetilde{r}_{i}^{k^{*}}\left(M_{k^{*}}, x_{\left[M^{k}\right]}^{k^{*}}, t-t^{k^{*}}\right)
\end{aligned}
$$

and (6) follows from the property $S M_{-}$of $\widetilde{r}^{k^{*}}$.

Step 3. Converse statement

Now we fix a markovian, consistent method $r$ satisfying PFS and $S M_{-}$, and we must show that $r$ is a method described in Definition 4. This proof is done in three steps, and is similar to that of Theorem 2 in Moulin and Stong [2000].

In the first step we construct the standard of comparison $\succsim$. Consider first the binary relation $R$ on $\mathcal{N} \times \mathbb{N}$ :

$$
\left(i, x_{i}\right) R\left(j, x_{j}\right) \Leftrightarrow\left\{i=j \text { and } x_{i} \geq x_{j}\right\} \text { or }\left\{i \neq j \text { and } p_{j}\left(x_{i} e_{i}+x_{j} e_{j}\right)>0\right\}
$$

From CSY and $S M_{-}^{0}$ (i.e., $p_{i}(N, x)$ is non-increasing in $x_{i}$ ), we check that $R$ is "almost" transitive in the following sense. If $i, j$ and $k$ are all distinct:

$$
\begin{aligned}
\left\{\left(i, x_{i}\right) R\left(j, x_{j}\right) \text { and }\left(j, x_{j}\right) R\left(j, x_{j}^{\prime}\right)\right\} & \Rightarrow\left\{\left(i, x_{i}\right) R\left(j, x_{j}^{\prime}\right)\right\} \\
\left\{\left(i, x_{i}\right) R\left(i, x_{i}^{\prime}\right) \text { and }\left(i, x_{i}^{\prime}\right) R\left(j, x_{j}\right)\right\} & \Rightarrow\left\{\left(i, x_{i}\right) R\left(j, x_{j}\right)\right\} \\
\left\{\left(i, x_{i}\right) R\left(j, x_{j}\right) \text { and }\left(j, x_{j}\right) R\left(k, x_{k}\right)\right\} & \Rightarrow\left\{\left(i, x_{i}\right) R\left(k, x_{k}\right)\right\}
\end{aligned}
$$


The top two statements follow easily from $S M_{-}^{0}$. We check the bottom statement, a consequence of CSY. Set $x=x_{i} e_{i}+x_{j} e_{j}+x_{k} e_{k}$ and apply CSY to $r(N, x, 1)$ three times:

$$
\begin{aligned}
& p_{j}(x)=\left(1-p_{k}(x)\right) \cdot p_{j}\left(x_{i} e_{i}+x_{j} e_{j}\right) ; p_{k}(x)=\left(1-p_{i}(x)\right) \cdot p_{k}\left(x_{j} e_{j}+x_{k} e_{k}\right) \\
& p_{k}(x)=\left(1-p_{j}(x)\right) \cdot p_{k}\left(x_{i} e_{i}+x_{k} e_{k}\right)
\end{aligned}
$$

We prove the bottom statement by contradiction: assume $p_{j}\left(x_{i} e_{i}+x_{j} e_{j}\right)$ and $p_{k}\left(x_{j} e_{j}+x_{k} e_{k}\right)$ are both positive, whereas $p_{k}\left(x_{i} e_{i}+x_{k} e_{k}\right)=0$, and the above system is impossible.

Note that $R$ may not be transitive because chains alternating between two agents are possible:

$$
\left(i, x_{i}\right) R\left(j, x_{j}\right) R\left(i, x_{i}^{\prime}\right) R\left(j, x_{j}^{\prime}\right) R \ldots \text { with } x_{i}>x_{i}^{\prime}>\ldots \text { and } x_{j}>x_{j}^{\prime}>\ldots
$$

But (26) implies that these are the only inclusion minimal chains.

The standard of comparison $\succsim$ is the transitive closure of $R$. The indifference classes of $\succsim$ will be represented, as usual, by $\mathcal{M}$-intervals [a,b[.

We check next that the support of $p(N, x)$ is contained in $M(x)$, namely the set of agents $i$ such that $\left(j, x_{j}\right) \succsim\left(i, x_{i}\right)$ for all $i$. For simplicity we write $p(x)$ in lieu of $p(N, x)$.

Suppose $p_{i}(x)>0$. Then for all $k$ in $N \backslash i$, CSY implies $p_{i}(x)=(1-$ $\left.p_{k}(x)\right) \cdot p_{i}\left(x_{-k}\right)$ hence $p_{i}\left(x_{-k}\right)>0$. Repeating the argument, we find $p_{i}\left(x_{i} e_{i}+\right.$ $\left.x_{j} e_{j}\right)>0$ hence $\left(j, x_{j}\right) R\left(i, x_{i}\right)$ for all $j \in N \backslash i$. This means $i \in M(x)$ because $\succsim$ contains $R$.

Next we show that $p(N, x)$ depends only on $x_{[M(x)]}$. This amounts to showing:

$$
i \notin M(x) \Rightarrow p(x)=p\left(x+e_{i}\right) \text { for all } N, x \text { and } i
$$

If $x, x^{\prime}$ are such that $M(x)=M\left(x^{\prime}\right)$ and $x, x^{\prime}$ coincide on this subset, there exists $x^{\prime \prime}$ with $M\left(x^{\prime \prime}\right)=M(x)$ and the same projection on $M(x)$, and such that $x \leq x^{\prime \prime}, x^{\prime} \leq x^{\prime \prime}$. Thus repeated applications of (28) yield $p(x)=p\left(x^{\prime}\right)$.

To prove $(28)$, we note that $p_{i}(x)=0$ (the support of $p$ is contained in $M(x))$, hence $p_{i}\left(x+e_{i}\right)=0$ by $S M_{-}^{0}$. For any $j$ in $N \backslash i$, apply CSY twice:

$$
\begin{aligned}
p_{j}(x) & =\left(1-p_{i}(x)\right) \cdot p_{j}\left(x_{-i}\right)=p_{j}\left(x_{-i}\right) \\
p_{j}\left(x+e_{i}\right) & =\left(1-p_{i}\left(x+e_{i}\right)\right) \cdot p_{j}\left(x_{-i}\right)=p_{j}\left(x_{-i}\right)
\end{aligned}
$$

establishing (28). We summarize the findings of Step 3. The method $r$ is entirely determined by the standard $\succsim$ and its restrictions to each indifference class $[a, b[$ of $\succsim$ : for any urn $(N, x), p(N, x)$ is supported by $M(x)$ and determined by the restriction of $p$ to $I(x)$, namely $p\left(M(x), x_{[M(x)]}\right)$. 
Step 4. The case of indifference classes of type at least 3 .

Let the $M$-interval $[a, b[$ be an indifference class of $\succsim$ such that $|\mathcal{M}| \geq 3$. We show that for all distinct $i, j$ in $\mathcal{M}$ :

$$
a_{i} \leq x_{i}<b_{i}, a_{j} \leq x_{j}<b_{j} \Rightarrow 0<p_{i}\left(x_{i} e_{i}+x_{j} e_{j}\right)<1
$$

By construction $\left(j, x_{j}\right) \sim\left(i, x_{i}\right)$, but we need $\left(j, x_{j}\right) R\left(i, x_{i}\right)$ to conclude $p_{i}\left(x_{i} e_{i}+x_{j} e_{j}\right)>0$. Choose a third agent $k$ in $\mathcal{M}$, and set $x_{k}=a_{k}$. We have $\left(j, x_{j}\right) \succsim\left(k, x_{k}\right)$ and $\left(k, x_{k}\right) \succsim\left(i, x_{i}\right)$. We know from Step 3 that an inclusion minimal chain from $\left(j, x_{j}\right)$ to $\left(k, x_{k}\right)$ or from $\left(k, x_{k}\right)$ to $\left(i, x_{i}\right)$ takes the form (27). Linking two such chains and using (26) repeatedly, we find $\left(j, x_{j}\right) R\left(i, x_{i}\right)$ ! Exchanging the roles of $i$ and $j$ establishes $\left(i, x_{i}\right) R\left(j, x_{j}\right)$ and (29) is proven.

Our next observation is that for all $M \subseteq \mathcal{M}$, and all $(M, x)$ in $[a, b[$, the support of $p(M, x)$ is exactly $M$. This implies that for all $(N, x)$,if $I(x)$ is of type at least 3 , the support of $p(N, x)$ is exactly $M(x)$ (we have seen in Step 2 that it is always contained in $M(x))$.

Fix $(M, x)$ in $[a, b[$. If $|M|=2$, property (29) shows that the support of $p(x)$ is $M$. If $|M| \geq 3$, we take $i$ in the support of $p(x)$, and two more agents $j, k$ in M. CSY implies:

$$
\begin{aligned}
p_{i}(M, x) & =\left(1-p_{k}(M, x)\right) \cdot p_{i}\left(M \backslash k, x_{-k}\right) \\
p_{j}(M, x) & =\left(1-p_{k}(M, x)\right) \cdot p_{j}\left(M \backslash k, x_{-k}\right) \\
& \Rightarrow p_{i}\left(M \backslash k, x_{-k}\right)>0 \text { and } \frac{p_{j}(M, x)}{p_{i}(M, x)}=\frac{p_{j}\left(M \backslash k, x_{-k}\right)}{p_{i}\left(M \backslash k, x_{-k}\right)}
\end{aligned}
$$

Repeat the argument to eliminate successively all agents in $M \backslash\{i, j\}$ so that:

$$
\frac{p_{j}(M, x)}{p_{i}(M, x)}=\frac{p_{j}\left(x_{i} e_{i}+x_{j} e_{j}\right)}{p_{i}\left(x_{i} e_{i}+x_{j} e_{j}\right)}
$$

and the desired conclusion $p_{j}(M, x)>0$ follows from (29).

Step 5. Final argument

We are now ready to show that $r$ takes the form announced in Definition 4 on its indifference classes of type at least 3 .

Fix such a class, a $\mathcal{M}$-interval $\left[a, b\left[\right.\right.$, where each $b_{i}$ can be finite or infinite. By Step 4 , the restriction of $p$ to $[a, b[$ satisfies Positive Shares, therefore we can duplicate the argument in the proof of Theorem 1, statement ii. We find there exists for all $i \in \mathcal{M}$ a positive function $f_{i}\left(x_{i}\right)$ defined over $\left[a_{i}, b_{i}[\right.$ and such that:

$$
p_{i}(M, x)=\frac{f_{i}\left(x_{i}\right)}{\sum_{M} f_{j}\left(x_{j}\right)} \quad \text { for all }(M, x) \text { in }[a, b[
$$


By setting $f_{i}\left(b_{i}\right)=0$ in case $b_{i}$ is finite, the above equation now holds for any $x$ s.t. $a \leq x \leq b$ and $x \neq b$. By $S M_{-}^{0}, f_{i}$ is non-increasing.

We now develop equation (23), the application of CSY to the allocation of two units among three agents $1,2,3$ in $M$ with shares $x_{i}, a_{i} \leq x_{i}<b_{i}, i=1,2,3$. Note that the computation only involves $f_{i}\left(x_{i}\right)$ and $f_{i}\left(x_{i}+1\right)$, and so it unfolds exactly as in the proof of Theorem 1, establishing $f_{1}\left(x_{1}\right)-f_{1}\left(x_{1}+1\right)=f_{3}\left(x_{3}\right)-$ $f_{3}\left(x_{3}+1\right)$.

We have shown the existence of a non-negative slope $s$ such that $f_{i}\left(x_{i}\right)-$ $f_{i}\left(x_{i}+1\right)=s$ for all $x_{i}$ in $\left[a_{i}, b_{i}[\right.$. We distinguish two cases:

Case $1 . b$ is bounded: $b_{i}<+\infty$ for all $i \in \mathcal{M}$. From $f_{i}\left(b_{i}\right)=0$ and $f_{i}\left(b_{i}-\right.$ 1) $>0$, we get $s>0$ and $f_{i}\left(x_{i}\right)=s .\left(b_{i}-x_{i}\right)$ so $p$ is the proportional method restricted to $[a, b[$.

Case 2. $b$ is unbounded: $b_{i}=+\infty$ for some $i \in \mathcal{M}$. If $b_{1}=+\infty$ and $b_{2}$ is finite, we get $f_{1}\left(x_{1}\right)-f_{1}\left(x_{1}+1\right)=f_{2}\left(b_{2}-1\right)>0$, which contradicts $f_{1}\left(x_{1}\right)>0$ for $x_{1}$ large enough. Therefore $b_{i}=+\infty$ for all $i \in \mathcal{M}$.

We claim that $\mathcal{M}=\mathcal{N}$ and that the $\mathcal{N}$-class $[a,+\infty[$ is the largest of $\succsim$. Suppose $\left[a,+\infty^{\mathcal{M}}\right.$ [ is not the largest $\succsim-$ indifference class; this can only happen if $\mathcal{M} \neq \mathcal{N}$. We can pick agent 1 inside $\mathcal{M}$, and agent 2 outside $\mathcal{M}$, with a share $x_{2}$ s.t. $\left(2, x_{2}\right) \succ(1, a)$. The urn $a e_{1}+x_{2} e_{2}$ will be filled with 1 -balls only, in contradiction of PFS.

Suppose next $\left[a, \infty^{\mathcal{M}}\right.$ [is the largest indifference class and $\mathcal{M} \neq \mathcal{N}$. Now the urn $a e_{1}+x_{2} e_{2}$ will be filled with 2-balls only, for any $x_{2}$, again a contradiction of PFS.

In the $\mathcal{N}$-interval $\left[a,+\infty\right.$ [, the equation $f_{i}\left(x_{i}\right)-f_{i}\left(x_{i}+1\right)=s$ together with $f_{i}\left(x_{i}\right)>0$ implies that $f_{i}$ is constant, for all $i$, therefore the restriction of $p$ to this interval is a fixed chances method, and the proof of Theorem 2 is complete.

\section{Step 6. Corollary to Theorem 2}

If $\succsim$ is symmetric, all indifference classes are symmetric of type $|\mathcal{N}| \geq 3$. Therefore a class $\left[a, b\left[\right.\right.$ is either bounded or has $b_{i}=+\infty$ for all $i \in \mathcal{M}$. This is the only place in the proof of Theorem 2 where PFS was used. The rest of the proof is routine.

\section{A generalization of Theorem 1}

By imitating the previous proof techniques, we can describe all the marko-

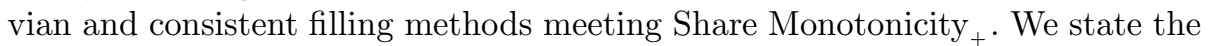
result without proof. Each such method is associated with a standard of comparison $\succsim$ (Definition 3 ) with only three possible types of indifference classes $I$ :

- a singleton $\mathcal{M}$-class, $I=[a]$, where $|\mathcal{M}| \geq 3$

- an unbounded $\mathcal{M}$-class $I=[a,+\infty[$, where $|\mathcal{M}| \geq 3$

- a class of type 2 or 1. 
For any urn $(N, x)$, the probability distribution $p(N, x)$ is concentrated on the set $Q(x)$ of agents $i$ such that $\left(i, x_{i}\right) \succsim\left(j, x_{j}\right)$ for all $j$. The restriction of $p$ to an unbounded class $[a,+\infty[$ (with $|\mathcal{M}| \geq 3$ ) is either a fixed chances or a PolyaEggenberger method. The restriction of $p$ to a class of type 2 meets $S M_{+}^{0}$ (that is, $p_{i}\left(x_{i} e_{i}+x_{j} e_{j}\right)$ is non-decreasing in $\left.x_{i}\right)$. If $I(x)$, the highest indifference class among $\left(i, x_{i}\right)$, is a singleton, the filling process is a "winner-takes-all": whoever among $Q(x)$ receives the first unit, will receive all the subsequent units as well, because $Q\left(x+e_{i}\right)=\{i\}$ whenever $i \in Q(x)$.

The family of methods just described is precisely characterized by the combination M, CSY and $\mathrm{SM}_{+}$. These methods can be loosely described as mixing winner-takes-all, Polya-Eggenberger, fixed chances, and methods involving two agents. Yet the pattern linking these various components is too complex to allow a more intuitive description of the family.

Nevertheless, the symmetric subfamily (i.e., M, CSY, SM + and ETE) is simple. All indifference classes are either a singleton or unbounded and there is an integer $k^{*}, k^{*}=0,1, \ldots$ such that for all urn $(N, x)$ :

if $x_{i} \leq k^{*}-1$ for some $i, p(N, x)$ is uniform over $\arg \max _{i} x_{i}$

if $x_{i} \geq k^{*}$ for all $i, p(N, x)$ is the equal chances or a symmetric P.E. method.

These methods are a hybrid of the symmetric winner-takes-all if some shares are below the threshold $k^{*}$, and one of the symmetric methods of Theorem 1 when no share is below $k^{*}$.

\section{APPENDIX 3: PROOF OF THEOREM 3}

Step 1.

We describe the consequences of $M+I T$.

By the markovian property, the urn filling is characterized by the probabilities $p_{i}(N, x)$. However by IT, $p_{i}(N, x)$ depends only on $i, x_{i}$, and $x_{N}$. Write it as $p_{i}(N, x)=\rho_{i}\left(x_{i}, x_{N}\right)$. Suppose $|N| \geq 3$, then applying IT to the coalition $\{i, j\}$ shows that

$$
\rho_{i}\left(x_{i}+1, x_{N}\right)-\rho_{i}\left(x_{i}, x_{N}\right)=\rho_{j}\left(1, x_{N}\right)-\rho_{j}\left(0, x_{N}\right) .
$$

Since this holds for all $i$ and $j$ in $N$ and all $x_{i}<N$, we see that $p_{i}(N, x)=$ $\rho_{i}\left(x_{i}, x_{N}\right)$ are all linear in $x_{i}$ with the same slope $\delta=\rho_{j}\left(1, x_{N}\right)-\rho_{j}\left(0, x_{N}\right)$ which is independent of $j$. There are three slightly different cases, positive slope, zero slope or negative slope. We can combine these by saying that a method satisfying $M$ and IT has

$$
p_{i}(N, x)=\frac{a\left(N, x_{N}\right) x_{i}+\lambda_{i}\left(N, x_{N}\right)}{\left.a\left(N, x_{N}\right) x_{N}+\lambda\left(N, x_{N}\right)\right)} .
$$


characterized by a set of non-negative weights $\lambda_{i}\left(N, x_{N}\right)$ and a "slope" $a\left(N, x_{N}\right)$ which takes values in $\{-1,0,1\}$ for each $N \subset \mathcal{N}$ with $|N| \geq 3$ and each $x_{N} \geq 1$. If $a\left(N, x_{N}\right)=1$, then $\lambda_{i}\left(N, x_{N}\right)$ can be any non-negative weights. If $a\left(N, x_{N}\right)=$ -1 , then we must have $\lambda_{i}\left(N, x_{N}\right) \geq x_{N}$ for all $i$. If $a\left(N, x_{N}\right)=0$, then $\lambda\left(N, x_{N}\right)$ is only defined up to a scalar multiple and we must have $\lambda_{N}\left(N, x_{N}\right)>0$. Note that for fixed $N$ (with $|N| \geq 3$ ) and $x_{N} \geq 1, a$ and $\lambda$ determine $p_{i}(N, x)$ and vice versa. If $x_{N}=0$, then the $p_{i}$ still have the form above, however $p_{i}$ does not depend on and hence does not determine $a(N, 0)$. If $|N|=2$, then IT is vacuous and $p_{i}$ may not have the form above. However if it does have this form we will continue to use the same notation.

Step 2.

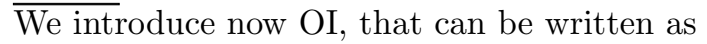

$$
p_{i}(x) p_{j}\left(x+e_{i}\right)=p_{j}(x) p_{i}\left(x+e_{j}\right)
$$

and plugging in the form

$$
p_{i}(x)=\frac{a\left(x_{N}\right) x_{i}+\lambda_{i}\left(x_{N}\right)}{a\left(x_{N}\right) x_{N}+\lambda_{N}\left(x_{N}\right)}
$$

we get

$x_{i}\left(a\left(x_{N}\right) \lambda_{j}\left(x_{N}+1\right)-a\left(x_{N}+1\right) \lambda_{j}\left(x_{N}\right)\right)+x_{j}\left(a\left(x_{N}+1\right) \lambda_{i}\left(x_{N}\right)-a\left(x_{N}\right) \lambda_{i}\left(x_{N}+1\right)\right)+$

$$
\left(\lambda_{i}\left(x_{N}\right) \lambda_{j}\left(x_{N}+1\right)-\lambda_{i}\left(x_{N}+1\right) \lambda_{j}\left(x_{N}\right)\right)=0 .
$$

Since $|N| \geq 3$, we can take $x_{i}=x_{j}=0$ giving

$$
\lambda_{i}\left(x_{N}\right) \lambda_{j}\left(x_{N}+1\right)=\lambda_{i}\left(x_{N}+1\right) \lambda_{j}\left(x_{N}\right)
$$

for all $i, j \in N$ and $x_{N} \geq 0$. Summing over $j \in N$ gives

$$
\lambda_{i}\left(x_{N}\right) \lambda_{N}\left(x_{N}+1\right)=\lambda_{i}\left(x_{N}+1\right) \lambda_{N}\left(x_{N}\right) .
$$

If $x_{N} \geq 1$, we can take $x_{i}=1$ and $x_{j}=0$, hence

$$
a\left(x_{N}\right) \lambda_{j}\left(x_{N}+1\right)=a\left(x_{N}+1\right) \lambda_{j}\left(x_{N}\right)
$$

for all $j \in N$ and $x_{N} \geq 1$. Summing this over $j$ gives

$$
a\left(x_{N}\right) \lambda_{N}\left(x_{N}+1\right)=a\left(x_{N}+1\right) \lambda_{N}\left(x_{N}\right) .
$$

Consider the three cases for $a$ separately. If $a\left(x_{N}\right)=0$, then $\lambda_{N}\left(x_{N}\right)>0$ and hence $a\left(x_{N}+1\right)=0$. Conversely if $a\left(x_{N}+1\right)=0$, then $\lambda_{N}\left(x_{N}+1\right)>0$ 
and hence $a\left(x_{N}\right)=0$. Thus if $a\left(x_{N}\right)=0$ for some $x_{N}$, then $a\left(x_{N}\right)=0$ for all $x_{N}$. Further since

$$
\lambda_{i}\left(x_{N}\right) \lambda_{N}\left(x_{N}+1\right)=\lambda_{i}\left(x_{N}+1\right) \lambda_{N}\left(x_{N}\right)
$$

we see that (modulo an irrelevant rescaling) $\lambda\left(x_{N}\right)$ is independent of $x_{N}$. Thus the method is a fixed chances method.

If one of $a\left(x_{N}\right)$ and $a\left(x_{N}+1\right)$ is 1 and the other is -1 , then we must have $\lambda_{N}\left(x_{N}+1\right)=0=\lambda_{N}\left(x_{N}\right)$. However if $a\left(x_{N}\right)=-1$, then $\lambda_{N}\left(x_{N}\right) \geq|N| x_{N}>$ 0 . Thus in either case we get a contradiction. Thus we see in any case that $a\left(x_{N}\right)=a$ is constant.

If $a= \pm 1$, then $\lambda_{j}\left(x_{N}+1\right)=\lambda_{j}\left(x_{N}\right)$ for all $j \in N$ and $x_{N} \geq 1$. Thus we conclude that $\lambda\left(x_{N}\right)$ is independent of $x_{N}$ for $x_{N} \geq 1$. If $a=-1$, we must have $\lambda_{j}\left(x_{N}\right) \geq x_{N}$ and hence a contradiction. Thus $a \neq-1$. If $\lambda_{N}\left(x_{N}\right) \neq 0$, then as for the case $a=0$, we see that $\lambda\left(x_{N}\right)$ is constant and the filling method is a PE method. If $\lambda_{N}\left(x_{N}\right)=0$, then $\lambda_{j}\left(x_{N}\right)=0$ for all $j \in N$ and all $x_{N} \geq 1$. However, we see that $\lambda(0)$ is arbitrary. Thus the method is quasi-proportional. This completes the proof.

\section{APPENDIX 4: Proof of Theorem 4}

Consider the bayesian urn-filling method associated to a measure $\pi$ described in terms of its sequence of moments $M(a)$. For any sequence $\sigma=\left\{i_{1}, i_{2}, \ldots, i_{t}\right\}$ with $\sum_{k=1}^{t} e_{i_{k}}=y-x$ one easily computes that proba $\{\sigma$ under $r$ at $(N, x)\}=$ $M(y) / M(x)$ is independent of permutations of $\sigma$. Hence such a method satisfies OI. Since $M(a)>0$ for all $a$ it also clearly satisfies PS and the weaker condition PFS. $\mathrm{SM}_{+}$is easier to prove from the alternate description of the bayesian method, so we will first check that the two are equivalent.

If we view $\pi=\pi_{0}$ as our prior distribution on the probabilities $\left(p_{i}\right)_{i \in N}$ of the various colors occurring, then $\pi_{x}$ is the posterior distribution after having observed $x_{i}$ occurrences of color $i$. Thus whenever we have $y_{i}$ balls in the urn of color $i$, we have the posterior distribution $\pi_{y}$ regardless of what combination of initial claims and balls added brought us to $y$. Thus at $(N, x)$ the probability that next ball added will be of color $i$ is $p_{i}(N, x)=E_{\pi_{x}}\left(p_{i}\right)=M\left(x+e_{i}\right) / M(x)$, and the two descriptions agree. Let $F_{x}^{(i)}(p)=\operatorname{proba}\left\{p_{i} \leq p\right.$ under $\left.\pi_{x}\right\}$ be the marginal distribution function of $p_{i}$ under the probability $\pi_{x}$. As far as agent $i$ is concerned, he will receive a probability of getting each ball with some probability $p_{i}$ chosen according to the distribution function $F_{x}^{(i)}$. Thus to show $\mathrm{SM}_{+}$it suffices to show that $F_{x+e_{i}}^{(i)}$ is stochastically greater than $F_{x}^{(i)}$. Since $\int_{0}^{p} \rho d F_{x}^{(i)}(\rho) \leq p F_{x}^{(i)}(p)$ and $\int_{p}^{1} \rho d F_{x}^{(i)}(\rho) \geq p\left(1-F_{x}^{(i)}(p)\right)$ we have

$$
F_{x+e_{i}}^{(i)}(p)=\frac{\int_{0}^{p} \rho d F_{x}^{(i)}(\rho)}{\int_{0}^{1} \rho d F_{x}^{(i)}(\rho)}=\frac{\int_{0}^{p} \rho d F_{x}^{(i)}(\rho)}{\int_{0}^{p} \rho d F_{x}^{(i)}(\rho)+\int_{p}^{1} \rho d F_{x}^{(i)}(\rho)}
$$




$$
\leq \frac{p F_{x}^{(i)}(p)}{p F_{x}^{(i)}(p)+p\left(1-F_{x}^{(i)}(p)\right)}=F_{x}^{(i)}(p),
$$

and stochastic dominance follows.

For the converse, suppose we have an urn-filling method which meets $\mathrm{M}$ and OI. Suppose we start with initial claims of 0 and add balls successively. Let $X_{t}$ be the color assigned to ball $t$. By OI, the sequence $X_{t}$ is exchangeable therefore by de Finetti's Theorem (Theorem 35.10 p. 473 of Billingsley [1995]), there is a probability measure $\pi$ on $\Sigma(N)$ such that the colors $X_{t}$ are independently chosen with probabilities $\left(p_{i}\right)_{i \in N}$, and the $\left(p_{i}\right)_{i \in N}$ chosen randomly according to $\pi$. To complete the proof that the urn-filling method is bayesian, we need only show that $\pi(\stackrel{\circ}{\Sigma}(N))>0$. This follows quickly from OI and PFS. Because of Order Independence, positive share at some future time PFS is equivalent to positive share immediately PS. Therefore there is positive probability that the first $|N|$ balls will be distributed one each to each of the agents, i.e.,

$$
\int_{\Sigma(N)} \prod_{i \in N} p_{i} d \pi(p)>0
$$

and hence $\pi(\stackrel{\circ}{\Sigma}(N))>0$.

From this discussion we also get a complicated characterization of urn-filling methods satisfying $\mathrm{M}$ and OI. For any initial set of claims $x$ we can look at the sequence $X_{t}$ of colors assigned to the successive balls. Again this sequence is exchangeable and de Finetti's theorem implies that there is a probability measure $\pi_{x}$ on $\Sigma(N)$ such that the colors $X_{t}$ are independently chosen with probabilities $\left(p_{i}\right)_{i \in N}$, where $\left(p_{i}\right)_{i \in N}$ are chosen according to $\pi_{x}$. If $E_{\pi_{x}}\left(p_{i}\right)=$ $\int_{\Sigma(N)} p_{i} d \pi_{x}(p)>0$, then there is positive probability that we will add a ball of color $i$ to obtain an urn containing $x+e_{i}$. Hence we must have

$$
\pi_{x+e_{i}}(A)=\frac{\int_{A} p_{i} d \pi_{x}(p)}{\int_{\Sigma(N)} p_{i} d \pi_{x}(p)} .
$$

However if $\int_{\Sigma(N)} p_{i} d \pi_{x}(p)=0$, then there is no direct relationship between $\pi_{x}$ and $\pi_{x+e_{i}}$. Such an urn-filling method could be referred to as quasi-bayesian, since for any set of initial claims it behaves like a bayesian method, but different initial claims may lead to unrelated bayesian methods.

\section{APPENDIX 5: $M+C S Y$}

One can give a very complicated description of all urn-filling methods satisfying M and CSY. The main invariant of such method is an ordering $\succsim$ on $\mathcal{N} \times \mathbb{N}$. For a finite set of agents $N$ and vector of claims $x$, let $M(x) \subset N$ be the set of agents $i$ such that $\left(i, x_{i}\right)$ is maximal under $\succsim$ and let $y$ be the projection of $x$ onto $M(x)$. At $(N, x)$ the only agents who have nonzero probability of 
receiving the next unit are those in $M(x)$ and further $p_{i}(N, x)=p_{i}(M(x), y)$ depends only on $y$. For an equivalence class $I \subset \mathcal{N} \times \mathbb{N}$ of $\succsim$, let $\mathcal{M}(I)$ be the set of all agents represented by $I$ and call $|\mathcal{M}(I)|$ the type of $I$. Then the possible equivalence classes are as follows:

(1) An equivalence class of type 1 is necessarily a singleton.

(2) An equivalence class of type 2 may be arbitrary and on this class the urnfilling method can be any markovian method.

(3) An equivalence class of type at least 3 may be of one of four types.

(3i) Winner-takes-all. The equivalence class $I$ consists of a single pair $\left(i, x_{i}\right)$ for each $i \in \mathcal{M}(I)$ and we have $\left(i, y_{i}\right) \succ\left(i, x_{i}\right)$ for all $y_{i}>x_{i}$. In this case there is some set of positive weights $\lambda_{i}$ for $i \in \mathcal{M}(I)$. If $M \subset \mathcal{M}(I)$ and $x$ is the $M$-vector with entries $x_{i}$, then $p_{i}(M, x)=\lambda_{i} / \lambda_{M}$ and the agent who receives that first unit will receive all subsequent units.

(3ii) Equalizing. There is a finite integer $s \geq 1$ and the equivalence class $I$ consists of a union of disjoint chains with constant increment $s,\left(i, a_{i}\right),\left(i, a_{i}+\right.$ $s), \ldots\left(i, b_{i}-s\right)$ and we have $\left(i, a_{i}\right) \succ\left(i, b_{i}\right)$ and $\left(i, y_{i}\right) \succ\left(i, a_{i}\right)$ for all $a_{i}<y_{i}<b_{i}$ which are not congruent to $a_{i} \bmod s$. Note that there may be more than one such chain for each agent $i$. Suppose $x$ is a vector of claims supported on $M \subset \mathcal{M}(I),\left(i, x_{i}\right) \in I$ for all $i$, and $\left(i, a_{i}\right), \ldots,\left(i, b_{i}-s\right)$ is the chain containing $\left(i, x_{i}\right)$, then

$$
p_{i}(M, x)=\frac{b_{i}-x_{i}}{b_{M}-x_{M}} .
$$

(3iii) PE. There is an integer $s \geq 1$ and for each agent $i \in \mathcal{M}(I)$ there is a nonnegative integer $a_{i}$ such that the equivalence class $I$ consists of all pairs $\left(i, a_{i}+k s\right)$ for $k \geq 0$. Furthermore $\left(i, y_{i}\right) \succ\left(i, a_{i}\right)$ for any $y_{i}>a_{i}$ and not of the form $a_{i}+k s$. In this case there is a vector $\lambda$ of positive weights supported on $\mathcal{M}(I)$ and if $x$ is a vector of claims supported on $M \subset \mathcal{M}(I)$ such that $\left(i, x_{i}\right) \in I$ for all $i \in M$, then

$$
p_{i}(M, x)=\frac{x_{i}-a_{i}+\lambda_{i}}{x_{M}-a_{M}+\lambda_{M}} .
$$

(3iv) fixed chances. There is an integer $s \geq 1$ and for each agent $i \in \mathcal{M}(I)$ there is a nonnegative integer $a_{i}$ such that the equivalence class $I$ consists of all pairs $\left(i, a_{i}+k s\right)$ for $k \geq 0$. Furthermore $\left(i, y_{i}\right) \succ\left(i, a_{i}\right)$ for any $y_{i}>a_{i}$ and not of the form $a_{i}+k s$. In this case there is a vector $\lambda$ of positive weights supported on $\mathcal{M}(I)$ and if $x$ is a vector of claims supported on $M \subset \mathcal{M}(I)$ such that $\left(i, x_{i}\right) \in I$ for all $i \in M$, then

$$
p_{i}(M, x)=\frac{\lambda_{i}}{\lambda_{M}}
$$

Note that in cases (3ii), (3iii), and (3iv), if an agent receives one ball, then he will receive the next $s-1$ balls as well.

One can also give a complete characterization of all urn-filling methods which satisfy the Markovian Property, Consistency and Order Independence. From 
the remarks in Appendix 4, it is easy to check that Order Independence implies Share Monotonicity+. In the opposite direction, a method which satisfies M, $\mathrm{CSY}$ and $\mathrm{SM}_{+}$is a combination of winner-takes-all, Polya-Eggenberger, fixed chances and methods involving 2 agents (Appendix 2). However winner-takesall, Polya-Eggenberger, and fixed chances methods all satisfy Order Independence. After a little analysis of the 2 agent case, one sees that a method satisfying M, CSY and OI is exactly a method satisfying $\mathrm{M}+\mathrm{CSY}+\mathrm{SM}_{+}$with the added restriction that on each equivalence class of type 2 the urn-filling method must be bayesian.

\section{APPENDIX 6: $M+C S Y+I T$}

Consider the generalization of the PE methods (7) or the fixed chances methods (8) where some of the weights $\lambda_{i}$ can be zero: the distribution $p(N, x)$ is well-defined whenever $N$ intersects the support of $\lambda+x$ or of $\lambda$ (for the cases of $\mathrm{PE}$ and fixed chances methods respectively). When it does not, we can use a different set of non-negative fixed chances to distribute the next unit. This leads to the following concept of nested fixed weights.

Definition 5 Given an ordering $\succsim$ of $\mathcal{N}$ (complete, transitive) and a set of positive weights $\mu_{i}$ for each $i \in \mathcal{N}$, we define the nested set of weights $\pi^{(\succsim, \mu)}$ associating to every finite subset $N$ of $\mathcal{N}$ the following set of convex weights:

$$
\begin{aligned}
\pi_{i}^{(\succsim, \mu)}(N) & =\frac{\mu_{i}}{\mu_{N}^{*}} \text { if } i \in N^{*}=\arg \max (\succsim, N) \\
& =0 \quad \text { if } i \notin N^{*}
\end{aligned}
$$

Note that the next set of weights is unchanged if we multiply all numbers $\mu_{i}$ in an indifference class of $\succsim$ by a positive factor.

Definition 6 Given a nested set of weights $\pi^{(\succsim, \mu)}$, we define three markovian urn-filling methods:

i) $\pi$ - fixed chances : for all $N, x, i$

$$
p_{i}(N, x)=\pi_{i}(N)
$$

ii) $\pi$-proportional : for all $N, x, i$

$$
p_{i}(N, x)=\frac{x_{i}}{x_{N}} \text { if } x_{N}>0 ; p_{i}(N, 0)=\pi_{i}(N)
$$

iii) $\pi$-Polya-Eggenberger: for all $N, x, i$

$$
p_{i}(N, x)=\frac{\lambda_{i}+x_{i}}{\lambda_{N}+x_{N}} \text { if } x_{N}>0 ; p_{i}(N, 0)=\pi_{i}(N)
$$

where $\lambda$ is the truncation of $\mu$ to $\mathcal{N}^{*}=\arg \max (\succsim, \mathcal{N})$ :

$$
\lambda_{j}=\mu_{j} \text { if } j \in \mathcal{N}^{*} \text { and } \lambda_{j}=0 \text { otherwise }
$$


Notice that the $\pi$-P.E. method is defined only if there are some largest agents for $\succsim$ in $\mathcal{N}$, which is always true when $\mathcal{N}$ is finite. The fixed chances and P.E. methods ((8),(7)) correspond to the case where all agents of $\mathcal{N}$ are indifferent for $\succsim$.

We claim that all methods in Definition 6 meet IT and CSY. The claim is obvious for IT. We check that a $\pi-P$.E. or a $\pi$-proportional method $r$ is consistent.

Fix an urn $(N, x)$, two agents $i, j$ and check equation (2). If $x_{N \backslash j}>0$, both filling processes $r(N, x)$ and $r(N \backslash j, x)$ are a P.E. method with non-negative coefficient $\lambda_{k}$ (they are all zero for the proportional method): the proof that a P.E. method is consistent in Appendix 1 extends to the case of non-negative $\lambda_{k}$.

If $x_{N \backslash j}=0$ and $x_{j}>0$, all balls in $r(N, x)$ go to agent $j$ under the $\pi$-proportional method, hence (2) is trivially true. For a $\pi$-P.E. method, distinguish two cases: if $(N \backslash j) \cap \mathcal{N}^{*}=\emptyset$, again all balls in $r(N, x)$ go to agent $j$, if $(N \backslash j) \cap \mathcal{N}^{*} \neq \emptyset$, then $r(N, x)$ and $r(N \backslash j, x)$ are the P.E. methods with non-negative coefficients $\lambda_{k}$ (for instance $r(N \backslash j, 0)$ gives the first ball to $k$ with proba. $\lambda_{k} / \lambda_{N \backslash j}$, and so on).

If $x_{N}=0$, then $r(N, 0)$ and $r(N \backslash j, 0)$ only ever give balls to $N^{*}$ and $(N \backslash j)^{*}$. If $j \notin N^{*}$ he is left out of the distribution and (2) is trivial. If $j \in N^{*}$, then the $\pi$-proportional process $r(N, 0)$ gives all the balls to one agent in $N^{*}$ selected according to the distribution $\pi\left(N^{*}\right)$ and (2) follows easily. The $\pi-$ P.E. method $r(N, 0)$ does the same if $N^{*} \cap \mathcal{N}^{*}=\emptyset$, otherwise it is the P.E. method with (positive) coefficients $\lambda_{k}$ on $N^{*}$, and (2) follows.

We need one more definition before stating the characterization result. Suppose $\mathcal{N}$ is partitioned as $\mathcal{N}_{+} \cup \mathcal{N}_{-}$, and $r_{+}, r_{-}$are two filling methods defined respectively on $\mathcal{N}_{+}$and $\mathcal{N}_{-}$. The priority composition of $r_{+}$and $r_{-}$(with $\mathcal{N}_{+}$ having the higher priority) is the method filling an urn $(N, x)$ like $r_{-}$whenever $N \subseteq \mathcal{N}_{-}$, and like $r_{+}$whenever $N \cap \mathcal{N}_{+} \neq \emptyset$-in the latter case, the agents in $N \backslash \mathcal{N}_{+}$receive nothing- .

The priority composition respects $\mathrm{M}$ and CSY, that is, the composed method meets M, or CSY if both $r_{+}$and $r_{-}$do. Priority composition does not respect IT, except when $r_{+}$is a fixed chances method.

A filling method $r$ meets M, CSY and IT if and only it is the priority composition of a $\pi_{+}$-fixed chances method $\left(\mathcal{N}_{+}, r_{+}\right)$and a method $\left(\mathcal{N}_{-}, r_{-}\right)$that is a $\pi_{-}$-proportional or a $\pi_{-}$-P.E. method, or a 2 -person method. Note that we allow $\mathcal{N}_{+}$or $\mathcal{N}_{-}$to be empty.

We omit the proof for the sake of brevity.

Note that all above methods are OI (resp. $\mathrm{SM}_{+}$) provided OI (resp. $\mathrm{SM}_{+}$) holds for the low priority 2-person method (if any). In this sense OI and $\mathrm{SM}_{+}$ "almost" follow from the combination M, CSY and IT. 


\section{References}

[1] Aumann, R. J. and M. Maschler. 1985. "Game Theoretic Analysis of a Bankruptcy Problem from the Talmud," Journal of Economic Theory, 36, 195-213.

[2] Banker, R. 1981. "Equity Considerations in Traditional Full Cost Allocation Practices: An Axiomatic Perspective," Joint Cost Allocations, S. Moriarty, Ed., Oklahoma City: University of Oklahoma Press, 110-130.

[3] Billingsley, P. 1995. Probability and Measure, John Wiley and Sons, New York.

[4] Chun, Y. 1989. "A non-cooperative justification for egalitarian surplussharing," Mathematical Social Sciences, 17, 245-261.

[5] De Frutos, M. A., 1999. "Coalitional Manipulations in a Bankruptcy Problem," Review of Economic Design, 4, 255-272.

[6] Herrero, C., M. Maschler and A. Villar, 1999. "Individual rights and collective responsability: the rights egalitarian solution," Mathematical Social Sciences, 37, 59-77.

[7] Johnson, N. and S. Kotz, 1977. Urn Models and their applications, John Wiley and Sons, New York.

[8] Moulin, H. 1987. "Equal or Proportional Division of a Surplus, and Other Methods," International Journal of Game Theory, 16, 3, 161-186.

[9] Moulin, H. 2000a. "Priority Rules and Other Asymmetric Rationing Methods," Econometrica, 68, 3, 643-684.

[10] Moulin, H. 2000b. "The Proportional Random Allocation of Indivisible Units," forthcoming, Social Choice and Welfare.

[11] Moulin, H. and R. Stong, 2000. "Fair Queuing and other Probabilistic Allocation Methods," forthcoming, Mathematics of Operations Research.

[12] O'Neill, B. 1982. "A Problem of Rights Arbitration from the Talmud," Mathematical Social Sciences, 2, 345-371.

[13] Thomson, W. 1995. "Axiomatic Analyses of Bankruptcy and Taxation Problems: A Survey," mimeo, University of Rochester.

[14] Young, H. P. 1987. "On dividing an amount according to individual claims or liabilities," Mathematics of Operations Research, 1,2,3, 398-414.

[15] Young, H. P. 1988. "Distributive Justice in Taxation," Journal of Economic Theory, 48, 331-335 